\title{
UNA EVALUACIÓN DEL PASS-THROUGH EN LA ARGENTINA USANDO FUNCIONES IMPULSO RESPUESTA DE CUANTILES MULTIVARIADOS ${ }^{\circ \circ}$
}

\author{
AN EVALUATION OF PASS-THROUGH IN ARGENTINA \\ USING MULTIVARIATE QUANTILE IMPULSE RESPONSE \\ FUNCTIONS
}

Gabriel Montes-Rojas*

enviado: 03 marzo 2019 - aceptado: 19 julio 2019

\begin{abstract}
Resumen
Este trabajo implementa modelos econométricos de cuantiles multivariados para evaluar la heterogeneidad del pass-through del tipo de cambio a precios y producto en la Argentina 2004-2018. El nuevo análisis revela heterogeneidad en las respuestas ante un shock cambiario. Primero, los efectos de la media, calculados con un modelo VAR en la media, no resultan adecuados para el producto y la inflación. De hecho, los efectos calculados de esta manera se condicen con cuantiles extremos en el análisis propuesto en este trabajo. Segundo, los efectos de pass-through usando el tipo de cambio oficial sobre el producto y la inflación dependen mayormente de los cuantiles de cada variable y no parece haber efectos cruzados. Es decir, los efectos sobre el producto varían con los cuantiles del producto, pero no de la inflación. En el mismo sentido, los efectos sobre la inflación no dependen de los cuantiles del producto y sí de la misma inflación. Existen efectos cruzados, sin embargo, cuando usamos el tipo de cambio blue. En términos del pass-through a precios se observa que el traslado es mayor (menor) para menores (mayores) cuantiles de la

Montes Rojas, G. (2019). Una evaluación del pass-through en la Argentina usando funciones impulso respuesta de cuantiles multivariados. Estudios económicos, 36 (73), 145-189.

- Se agradecen los comentarios del editor Germán González y dos evaluadores anónimos por sus críticas constructivas. Los potenciales errores que quedasen son de mi exclusiva responsabilidad.

* CONICET-Universidad de Buenos Aires; Instituto Interdisciplinario de Economía Política de Buenos Aires. Buenos Aires, Argentina. Correo electrónico: gabriel.montes@fce.uba.ar
\end{abstract}


inflación. Tercero, tanto el producto como la inflación dependen de los cuantiles del tipo de cambio. Esto significa que ciertas dinámicas altas del tipo de cambio pueden producir dinámicas de alto pass-through (negativo para el producto, positivo para la inflación).

Código JEL: C13, C14, C42.

Palabras clave: pass-through, funciones impulso respuesta, Argentina, cuantiles multivariados.

\begin{abstract}
This article implements multivariate quantiles econometric models to evaluate the heterogeneity in price and product pass-through in Argentina 2004-2018. The novel analysis reveals response heterogeneity after an exchange rate shock. First, the mean effects computed using standard VAR techniques on the mean are not adequate for product and inflation. In fact, the effects computed this way are similar to those in extreme quantiles as estimated in this paper. Second, pass-through effects using the official exchange rate on inflation and product mainly depend on each variables' quantiles, and there are no cross effects. That is, the product effects vary with the product quantiles, but not those of inflation. Similarly, the effects on inflation do not depend on product but on inflation only quantiles. There are cross effects, however, when using the unofficial exchange rate. The pass-through effect on prices is higher (lower) for low (high) inflation quantiles. Third, both product and inflation depend on the exchange rate quantiles. Thus certain exchange rate dynamics may produce high pass-through dynamics (negative for product, positive for inflation).
\end{abstract}

Jel Code:C13, C14, C42.

Keywords: pass-through, impulse response functions, Argentina, multivariate quantiles. 


\section{INTRODUCCIÓN}

Se entiende como coeficiente o elasticidad de pass-through (o de traspaso o traslado) al impacto que posee una variación del tipo de cambio sobre los precios de una economía. Para la Argentina, el estudio de la magnitud y persistencia del pass-through juega un rol central en todo análisis de política macroeconómica (no solo monetaria) e incluso en estudios de crecimiento y desarrollo. En nuestro caso interesa el traslado al índice de precios al consumidor (IPC), es decir, sobre la inflación, y también sobre el nivel de actividad económica o producto. Este trabajo estudia la heterogeneidad en los efectos de pass-through sobre precios y producto usando una técnica novedosa de cuantiles multivariados. En particular, se busca evaluar que gama de posibilidades pueden ocurrir luego de un shock cambiario. En otras palabras, este trabajo busca estimar la distribución de los efectos macroeconómicos luego de un shock cambiario.

Los modelos de vectores autorregresivos (VAR) son usados comúnmente para explorar la dinámica de distintos efectos de shocks macroeconómicos sobre un sistema de variables. El modelo VAR provee técnicas estadísticas para la descripción, predicción e inferencia estructural de series de tiempo multivariadas. En particular, la técnica relacionada se define como funciones impulso respuesta (FIR), que corresponde al análisis dinámico de un shock a lo largo del tiempo. En nuestro caso, el principal interés es estudiar el cambio de un shock sobre el tipo de cambio, es decir, una devaluación o apreciación cambiaria, sobre el nivel de precios y la actividad económica. Mientras el estudio de las FIR en la media condensa información al promediar toda la heterogeneidad, los hacedores de política pueden estar interesados en toda la distribución de efectos. En particular, en la ocurrencia de eventos extremos que pueden causar una crisis y/o desencadenar ciertos comportamientos no deseados. El estudio de la gama de posibilidades que pueden ocurrir ante un determinado shock se usa en varios contextos financieros y macroeconómicos. Por ejemplo, las regulaciones bancarias y financieras en muchos países, donde ciertos agentes están obligados a reportar las distintas posiciones de pérdida que pueden ocurrir en casos poco probables pero posibles, es decir, el llamado Value-at-Risk o también el Expected Shortfall. Una entidad financiera debe estimar y reportar el 5\% Value-at-Risk que se correspondería con el valor de retorno de un activo o portfolio que ocurriría si la realización aleatoria correspondiese al 5\% percentil más bajo. Este evento ocurre de forma poco probable, de hecho $95 \%$ de las veces tendría un retorno mayor a ese valor, pero la ocurrencia es probable y de interés.

En nuestro caso, el traslado a precios o efecto sobre el producto ante una devaluación a apreciación del tipo de cambio tiene toda una serie de distintos efectos 
potenciales, de los cuales las FIR calculadas en base al modelo VAR sobre la media solo calculan el efecto promedio. Este trabajo estudia el pass-through sobre la base de modelos de cuantiles multivariados, que son una generalización de los modelos VAR que permiten estudiar toda la distribución de impactos potenciales (ej., precios y producto) ante un shock determinado (ej., shock en el tipo de cambio). La técnica econométrica denominada regresión por cuantiles ( $\mathrm{RC}$, quantile regression en inglés) es un método estadístico para estudiar el efecto de variables de control sobre los cuantiles condicionales de otra variable. Este método ofrece una estrategia para examinar cómo las variables de control afectan la centralidad, escala y forma de la distribución condicional de esta variable, así permitiendo que se explore la presencia de heterogeneidad en las respuestas dinámicas, incluyendo diferencias de efectos y asimetrías.

Sobre la base de la aplicación de los modelos RC a modelos multivariados, Montes-Rojas (2019) propone un modelo VAR para cuantiles (VARQ) que permite una modelización de la heterogeneidad de las funciones impulso respuesta por cuantiles (FIRQ). Imponiendo distintos cuantiles multivariados en las ecuaciones de las variables endógenas se puede analizar las distintas trayectorias de las variables ante un shock determinado, así generalizando el impacto sobre la media o promedio que se corresponde con el análisis tradicional de las FIR.

En el presente trabajo se estiman los modelos VAR y VARQ, de los cuales se desarrolla un análisis de FIR y FIRQ, respectivamente, usando un modelo simple de tres variables macroeconómicas: tipo de cambio (en primeras diferencias de logaritmos, oficial y blue), precios al consumidor (en diferencias de logaritmos, en base a una serie de precios empalmadas de IPC) y nivel de actividad (serie desestacionalizada y sin tendencia, usando un indicador de actividad industrial, EMAE) para el período enero 2004-agosto 2018. Un shock de una unidad en el tipo de cambio, en diferencias, es construido a partir de una descomposición estándar de Cholesky. El nuevo análisis revela heterogeneidad en las respuestas ante un shock cambiario. El presente ejercicio econométrico intenta cuantificar las distintas respuestas ante un mismo shock cambiario a través de la modelización de las distribuciones de las FIRQ. Cabe destacar que el presente artículo es una aplicación de las técnicas econométricas desarrolladas en Montes-Rojas (2017, 2019), pero cuya implementación en la evaluación del pass-through en la Argentina es original.

El trabajo se organiza de la siguiente manera. La sección 2 hace una breve revisión de la literatura de pass-through. La sección 3 presenta la teoría de cuantiles direccionados, la definición del modelo VARQ y la de las FIRQ. La sección 4 presenta los resultados para el caso argentino. La sección 5 presenta una discusión de los resultados obtenidos. 


\section{REVISIÓN DE LA LITERATURA DE PASS-THROUGH}

Un exhaustivo análisis de la literatura de pass-through puede verse en Menon (1995), Miller (2003), Palleja (2017) y Castiglione (2017); a su vez, este último autor, además de presentar la literatura reciente, analiza el caso argentino en detalle. A continuación, presentamos algunos trabajos relevantes, enfatizando la abundante evidencia de efectos asimétricos y heterogéneos que motivan el análisis econométrico desarrollado en este trabajo.

El coeficiente de pass-through puede analizarse bajo diferentes categorías entre las que se destacan su magnitud, velocidad y simetría. Por magnitud se entiende a la proporción de la variación en el tipo de cambio que se traslada a los precios bajo análisis, es decir, la elasticidad de un cambio en el tipo de cambio sobre los precios. Un análisis dinámico permite ver el horizonte temporal de los efectos, $\mathrm{y}$ en particular las diferencias entre los efectos de corto y largo plazo. Finalmente, la asimetría de este coeficiente está vinculada a las diferencias en la reacción de los precios ante las variaciones cambiarias. Un pass-through asimétrico puede estar vinculado a variaciones de diferente índole frente a depreciaciones o apreciaciones, llamadas asimetrías en la dirección, o bien a variaciones diferentes dependientes de la magnitud de las variaciones, llamadas asimetrías de tamaño (Aron et al., 2014). Caselli y Roitman (2016) encuentran abundante evidencia de no linealidades y asimetrías en países emergentes. Ver también Barhoumi (2006), Beirne y Bijsterbosch (2011) y Ghartey (2018)1.

Dentro de los principales hechos estilizados encontrados en la gran mayoría de los estudios se destaca una gran heterogeneidad de los coeficientes entre sectores productivos, países y regiones (Aron et al., 2014; Castiglione, 2017). En general, el pass-through es mayor en países emergentes que en países desarrollados (ver, por ejemplo, Ca'Zorzi et al., 2007; McCarthy, 2007; Choudhri y Hakura, 2006; Law, Satoh y Yoshimi, 2019).

La heterogeneidad de los pass-through también se observa dentro de un mismo país. Taylor (2000) propone que el coeficiente de traslado será menor en economías con menor inflación. En ambientes de baja inflación, los shocks inflacionarios generados por variaciones cambiarias no serán percibidos como persistentes por lo que no tenderían a ser trasladados a precios de forma completa o veloz. En este caso, menos inflación reduce el pass-through y esto nuevamente reduce las presiones inflacionarias ante shocks externos (ver también Edwards, 2006).

1 Se agradecen las citas a un evaluador anónimo. 


\section{MODELO ECONOMÉTRICO}

\section{II.1. Modelo VARQ}

Este trabajo utiliza un modelo de cuantiles multivariados que generaliza los modelos VAR para cuantiles, permitiendo así explorar la heterogeneidad en los efectos. Estos modelos multivariados se descomponen en una magnitud y una dirección (vectorial), sobre la base de los trabajos de Hallin, Paindaveine y Siman (2010), Paindaveine y Siman $(2011,2012)$, Carlier, Chernozhukov y Galichon (2016) y Montes-Rojas (2017).

Consideremos un proceso multivariado de $m$ variables, $Y_{t}=\left(Y_{1 t}, \ldots, Y_{m t}\right)^{\prime}$ $\in \mathrm{R}^{m}$. Consideremos además un vector $k \times 1$ de variables de control $\mathrm{X}_{t}$. Nuestro interés se centra en los contrales generados por el campo-sigma definido por $\left\{Y_{s}\right.$ : $\mathrm{s}<\mathrm{t}$ \} junto con la otra información disponible en $t$. Para el caso VARQ de orden $p, \mathrm{X}_{t}=\left(\mathrm{Y}_{t-1}, \ldots, \mathrm{Y}_{t-p}\right)^{\prime} \operatorname{con} k=m p$. Indexamos entonces los modelos VARQ con el orden los rezagos, $\operatorname{VARQ}(\mathrm{p})$.

Consideremos también el vector $\tau=\left(\tau_{1}, \ldots, \tau_{m}\right)$ como un índice de magnitudes de los cuantiles correspondientes a cada variable endógena. Montes Rojas (2019) considera un modelo VARQ como un modelo VAR en forma reducida indexado por $\tau$,

$$
\mathrm{Q}_{Y_{t}}\left(\tau \mid x_{t-1}=X_{t-1}\right)=\mathrm{B}(\tau) x_{t-1}+\mathrm{A}(\tau)
$$

donde $\mathrm{Q}$ es un vector $m \times 1$ que corresponde a los cuantiles multivariados de las $m$ variables endógenas, $\mathrm{B}(\tau)=\left(\mathrm{B}_{1}(\tau), \ldots, \mathrm{B}_{m}(\tau)\right)$ ' es una matriz $m \times k$ de coeficientes con $\mathrm{B}_{j}(\tau)$ para cada $\mathrm{j}=1, \ldots, \mathrm{m}$, vectores $k \times 1$ de coeficientes del elemento $j$ de $\mathrm{Y}, \mathrm{y} \mathrm{A}(\tau)$ un vector $m \times 1$. Entonces $\mathrm{Q}$ mapea el vector de cuantiles y los rezagos en los distintos valores que puede tomar la variable dependiente. El modelo propuesto en (1) se puede estimar usando $m$ regresiones por cuantiles univariadas de cada una de las $j=1,2, \ldots, m$ variables endógenas con respecto a todos los rezagos (propios y de las otras variables), cada una evaluada en el correspondiente componente del vector $\tau$.

Los modelos de cuantiles condicionales en series de tiempo se pueden interpretar en términos del ciclo de las variables (ver Koenker y Xiao, 2006; Galvao, Montes-Rojas y Park, 2013). Por ejemplo, un cuantil bajo del producto, condicio- 
nal en la evolución pasada del producto y de otras variables, se corresponde a una performance baja con respecto a su tendencia histórica. Es decir, en el cuantil bajo el producto está por debajo de lo que correspondería a lo esperado de acuerdo a los rezagos del modelo. Si lo pensamos en términos del ciclo económico, modelizar un cuantil bajo corresponde a estados recesivos del ciclo. Lo contrario sucede para un cuantil alto, que se correspondería a la fase expansiva del ciclo. En el modelo multivariado que estamos usando en este trabajo, lo mismo puede usarse para cada una de las $m$ ecuaciones en (1) y para cada correspondiente elemento en $\tau$.

Montes-Rojas (2017) desarrolla un modelo de cuantiles multivariados que dan lugar a un VAR en forma reducida, sobre la base de los trabajos de Hallin et al. (2010) y trabajos posteriores. Este marco propuesto permite desarrollar un modelo de vectores autorregresivos por cuantiles (Montes-Rojas, 2019), VARQ) el cual generaliza el modelo de Koenker y Xiao (2006) para series de tiempo multivariadas. El modelo corresponde a una solución de una serie de cuantiles con dirección vectorial prefijada que corresponden a una base ortonormal (es decir, vectores ortogonales entre sí que permiten generar todo el espacio de las variables). Cada dirección se corresponde a una variable endógena en el sentido de Hallin et al. (2010). Este modelo es equivalente al de la ecuación (1).

\section{II.2. Evaluación de shocks, funciones impulso respuesta}

Ramey (2016) define un shock como fuerzas primitivas exógenas no correlacionadas entre sí que deberían ser económicamente significativas (pp. 52-55). Los llamados shocks deben cumplir con las siguientes características: (1) deberían ser exógenas con respecto a las otras variables endógenas (y sus rezagos); (2) deberían no estar correlacionados con otros shocks exógenos; (3) deberían representar movimientos no anticipados en variables exógenas o acerca de futuros movimientos de las mismas. Ver Ramey (2016) y Stock y Watson (2016) para un repaso de la literatura al respecto.

En nuestro caso, la idea sería encontrar cómo definir y separar los shocks al tipo de cambio, es decir, cambios exógenos en el tipo de cambio. Siguiendo con la literatura de identificación de shocks monetarios usamos un VAR estructural para definir los shocks al tipo de cambio (ver la siguiente sección en la que se usa la identificación de Cholesky). 
Para la modelización de cuantiles multivariados se considera un shock como un efecto contrafactual $\delta \in \mathrm{R}^{m}$. Así se analiza la comparación del modelo condicional en $\mathrm{x}_{t}^{\delta}=\left(\mathrm{y}_{\mathrm{t}}+\delta, \mathrm{y}_{t-1} \ldots, \mathrm{y}_{t-p}\right)^{\prime}$ con aquel en $x_{t}=\left(\mathrm{y}_{t}, \mathrm{y}_{t-1} \ldots, \mathrm{y}_{t-\mathrm{p}}\right)^{\prime}$.

A continuación, se exponen la forma en que se construyen la FIRQ, siguiendo a Montes-Rojas (2019). Sea la FIRQ para $\tau_{1}$ en $t+1$ para un shock en $t$, de magnitud $\delta$, como

$$
\operatorname{FIRQ}_{1}\left(\tau_{1}, \delta \mid x_{t}\right)=\mathrm{Q}_{1}\left(\tau_{1} \mid x_{t}^{\delta}\right)-\mathrm{Q}_{1}\left(\tau_{1} \mid x_{t}\right),
$$

donde $Q_{1}$ es la predicción un período hacia adelante, evaluada en $\tau_{1}$.

Consideremos ahora la predicción en $t+2$, usando los cuantiles $\tau_{2}$. Notar que esta respuesta depende de aquella en $t+1$ y por ende $\tau_{1}$. Es decir, el efecto en $t+2$ deberá ser evaluado en $\left(\tau_{1}, \tau_{2}\right)$, definido como un sendero de cuantiles. Definamos entonces

$$
\operatorname{FIRQ}_{2}\left(\left(\tau_{1}, \tau_{2}\right), \delta \mid x_{t}\right)=\mathrm{Q}_{2}\left(\left(\tau_{1}, \tau_{2}\right) \mid x_{t}^{\delta}\right)-\mathrm{Q}_{2}\left(\left(\tau_{1}, \tau_{2}\right) \mid x_{t}\right)
$$

donde $\mathrm{Q}_{2}$ es la predicción dos períodos hacia adelante, evaluada en el sendero de cuantiles $\left(\tau_{1}, \tau_{2}\right)$.

Este procedimiento se puede generalizar para $h$ períodos hacia adelante, definiendo el siguiente sendero de cuantiles de $h$ períodos $\left(\tau_{1}, \tau_{2, \ldots,} \tau_{\mathrm{h}}\right)$, y definiendo

$$
\begin{aligned}
& \operatorname{FIRQ}_{h}\left(\left(\tau_{1}, \tau_{2}, \ldots, \tau_{h}\right), \delta \mid x_{t}\right)=Q_{h}\left(\left(\tau_{1}, \tau_{2}, \ldots, \tau_{h}\right) \mid x_{t}^{\delta}\right)- \\
& \mathrm{Q}_{h}\left(\left(\tau_{1}, \tau_{2}, \ldots, \tau_{h}\right) \mid x_{t}\right) .
\end{aligned}
$$

Cada sendero se corresponde a una cierta configuración de las variables. Por ejemplo, un sendero con un cuantil alto de la variable $j$, se puede interpretar como la FIRQ que correspondería a una performance de dicho cuantil condicional, donde siempre se condiciona en la evolución pasada de todas las variables. Así podemos modelar distintas configuraciones de las variables que darían lugar a distintas evoluciones de las $m$ variables endógenas. Además de las FIR nos interesa las FIR acumuladas, que se construyen sumando los efectos de los períodos intermedios. De esta manera definimos aFIR y aFIRQ como las funciones impulso-respuesta acumuladas para la media y para los cuantiles, respecivamente. 


\section{II.3. Distribución de la FIRQ}

El modelo de FIRQ permite evaluar la distribución de las respuestas a un shock en determinados horizontes temporales. De esta manera, podemos estudiar la probabilidad de ocurrencia de eventos extremos, como por ejemplo una gran recesión conjuntamente con una inflación alta, luego de un shock determinado. Para ello lo que necesitamos es simular distintos senderos de cuantiles con variables uniformes multivariadas, $\tau_{\mathrm{j}} \sim$ iid Uniforme $(0,1)^{\mathrm{m}}, j=1,2, \ldots, h$. Esta simulación se corresponde en realidad con una modelización de coeficientes aleatorios como en Koenker y Xiao (2006) pero aplicado al modelo multivariado.

La idea es que cada realización, en cada período de tiempo, depende de los cuantiles de todas las variables endógenas. La gama de posibilidades de ocurrencia en $t+j$, dada la información en $t$, depende de los distintos valores que pueden tener estos cuantiles en cada uno de los periodos $t+1, t+2, \ldots, t+j$. Simulando vectores de variables aleatorias independientes uniformes en cada período sucesivo se pueden evaluar todas esas posibilidades con igual probabilidad.

Usando un procedimiento similar podemos construir la distribución de la aFIRQ, las respuestas acumuladas. En este caso, las sumas de los efectos acumulados se simulan con vectores de variables uniformes independientes entre sí en cada caso intermedio.

\section{HETEROGENEIDAD EMPÍRICA DEL PASS-THROUGH EN LA ARGENTINA}

\section{III.1. Datos}

Se utilizan datos para el período enero 2004 a agosto 2018, 176 observaciones. Las series usadas son las siguientes:

- Índice de Precios al Consumidor (IPC), varias fuentes, base 1999=100. En este caso usamos una serie empalmada de distintas fuentes debido a que las estadísticas publicadas por INDEC (Instituto Nacional de Estadísticas y Censos) para el período 2007-2015 fueron desacreditadas oficialmente por el organismo. En particular, usamos la series histórica de 2004 hasta diciembre 2006, luego usamos de Ciudad de Buenos Aires enero 2007 hasta mayo 2016, y retomamos la serie del INDEC. 
- Estimador Mensual de Actividad Económica (EMAE), fuente: INDEC.

- Tipo de cambio nominal oficial promedio mensual, fuente: BCRA (http://www. bcra.gov.ar/pdfs/operaciones/com3500.xls).

- Tipo de cambio nominal blue promedio mensual, fuente: Ámbito Financiero (http://www.ambito.com/economia/mercados/dolar.asp).

III.2. Hechos estilizados: pass-through no condicional

La figura 1 reporta las series originales en logaritmo junto con la tendencia medida con el filtro de Hodrick-Prescot. La economía argentina se puede definir como una economía de media-alta inflación con un desaceleramiento de la economía real a partir de 2011. El tipo de cambio contiene menores variaciones con respecto a la tendencia que el resto de las series.

Sea $\mathrm{p}_{\mathrm{t}}$ el logaritmo del IPC, $\mathrm{r}_{\mathrm{t}}$ el logaritmo del tipo de cambio, e $\mathrm{y}_{\mathrm{t}}$ las desviaciones del producto (medido por el logaritmo del EMAE) con respecto a la tendencia.

Una primera aproximación a los efectos de pass-through se basa en las distribuciones de los ratios de cambios en los precios dado un determinado cambio en el tipo de cambio. Se computan los ratios de los cambios en los logaritmos contemporáneos, uno y dos períodos hacia adelante, para devaluaciones y apreciaciones, por separado. Es decir, se computan los ratios $\left(p_{t+j}-p_{t-1}\right) /\left(r_{t}-r_{t-1}\right)$ para distintos horizontes $\mathrm{j}$. En este caso, $\mathrm{j}=0$ es el cambio contemporáneo (mismo mes), $\mathrm{j}=1$ el mes siguiente, y $\mathrm{j}=2$ dos meses hacia adelante. En todos los casos se impone un valor mínimo a $\left|r_{t}-r_{t-1}\right|_{>} \epsilon$ para no dividir por un valor cercano a 0 . Este ratio constituye una estimación del pass-through a precios sin condicionar en la información disponible.

Los gráficos (a), (b), (e) y (f) que aparecen en la figura 2 reportan la distribución (estimada por el método de kernels) de los ratios, usando $\epsilon=.001$, para el tipo de cambio oficial (arriba) y el tipo de cambio blue (abajo), respectivamente. Las devaluaciones están asociadas en promedio con efectos positivos, o sea, traslado a precio positivo. Sin embargo, la magnitud de la elasticidad tiene menos heterogeneidad a medida que aumenta $j$, indicando que el efecto dos períodos hacia adelante es más estable que el contemporáneo. Por otro lado, las apreciaciones también tienen un efecto positivo sobre los precios, es decir, las apreciaciones cambiarias (denominador negativo) también se reflejan en aumentos de los precios con el mismo grado de heterogeneidad (el ratio es entonces también negativo). En 

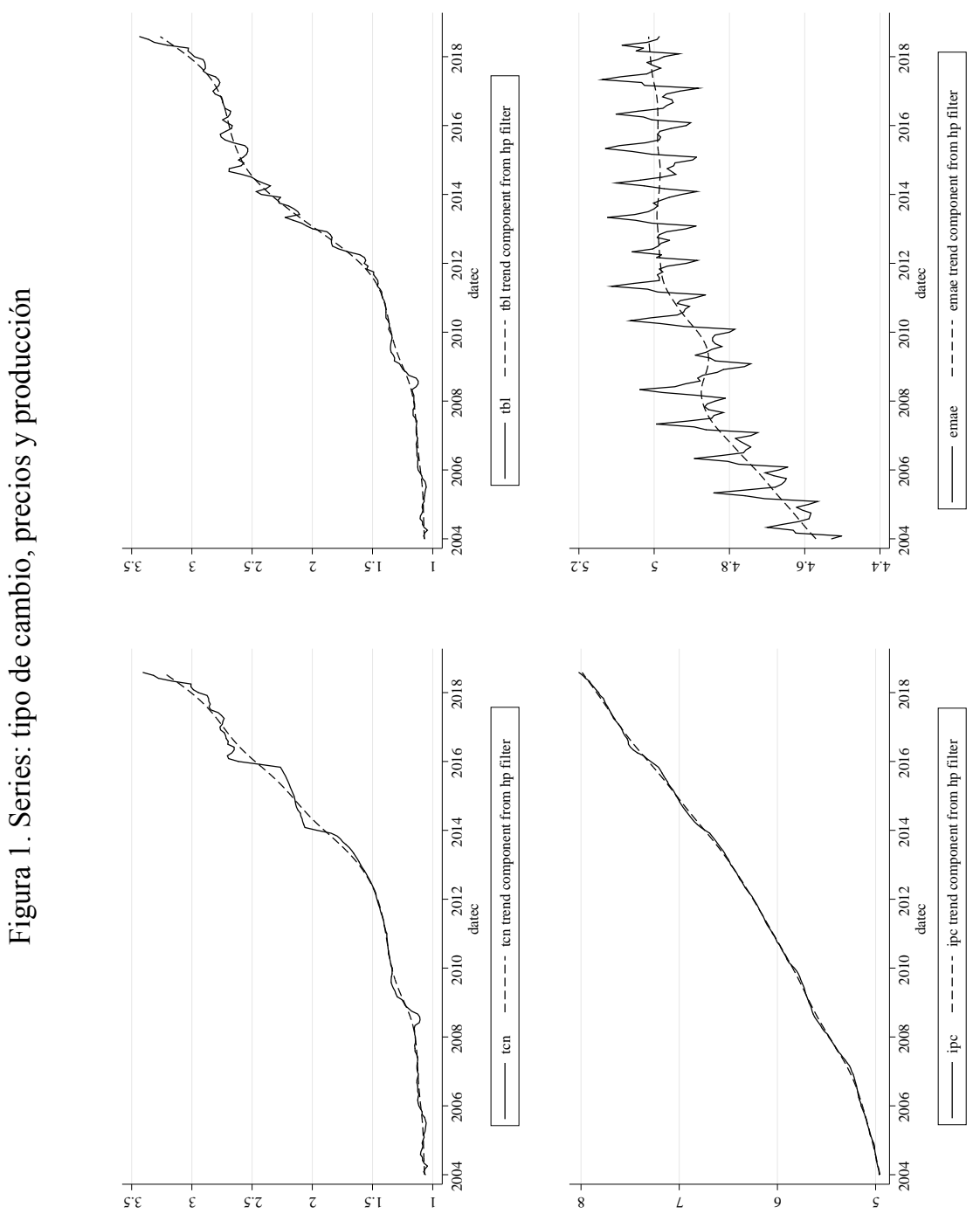
promedio pareciera que el efecto dos períodos hacia adelante se centran en una elasticidad de pass-through a precios menor a 2, asimétrica para depreciaciones y apreciaciones, es decir, en ambos casos los precios se incrementan.

Los gráficos $(\mathrm{c}),(\mathrm{d}),(\mathrm{g})$ y $(\mathrm{h})$ reportan los ratios a través del tiempo, también separado para depreciaciones y apreciaciones. En este caso usamos $\epsilon=.01$. Los efectos confirman los signos y la estabilidad en ambos casos para $\mathrm{j}=2$ y los distintos efectos. No se observan grandes diferencias si se usa el tipo de cambio oficial o el blue.

Consideremos ahora los efectos de pass-through sobre el producto. Es decir, se computan los ratios $\left(y_{t+j}-y_{t-1}\right) /\left(r_{t}-r_{t-1}\right)$ para distintos horizontes $j$, separando apreciaciones y depreciaciones. Los gráficos en la figura 3 muestran estos efectos para el tipo de cambio oficial y el blue. Los gráficos (a), (b), (e) y (f) que aparecen en la figura 3 reportan la distribución de los ratios usando $\epsilon=.001$, mientras que los gráficos (c), (d), (g), y (h) reportan los ratios a través del tiempo, con $\epsilon=.01$. No se observa un efecto claro para los efectos contemporáneos, $\mathrm{j}=0$, pero sí una distribución bimodal para $\mathrm{j}=1,2$. Es decir, tanto depreciaciones del peso como apreciaciones pueden llevar a crecimiento o reducción del producto.

\section{III.3. Modelo econométrico}

Los resultados de la sección anterior sugieren que un único modelo, VAR en la media, no puede capturar toda la heterogeneidad que puede ocurrir luego de un shock en el tipo de cambio. Consideremos ahora un modelo VAR de 3 variables: $\pi_{\mathrm{t}}$, primera diferencia del logaritmo del IPC, $\rho_{\mathrm{t}}$, primera diferencia del logaritmo del tipo de cambio, e $y_{t}$ producto desestacionalizado en desviaciones con respecto a la tendencia. Los datos son para el período enero-2004 a agosto-2018 en periodicidad mensual. Para estas transformaciones de las variables originales se rechaza la presencia de raíz unitaria en todos los casos. Para este caso entonces tenemos Yt $=\left(\mathrm{y}_{\mathrm{t}}, \pi_{\mathrm{t}}, \rho_{\mathrm{t}}\right)^{\prime}$. La figura 4 presenta las variables usadas para este modelo econométrico. Utilizando el criterio de información bayesiano en la media de las 3 variables usamos un modelo con un rezago (VAR(1)) para todos los modelos implementados. 

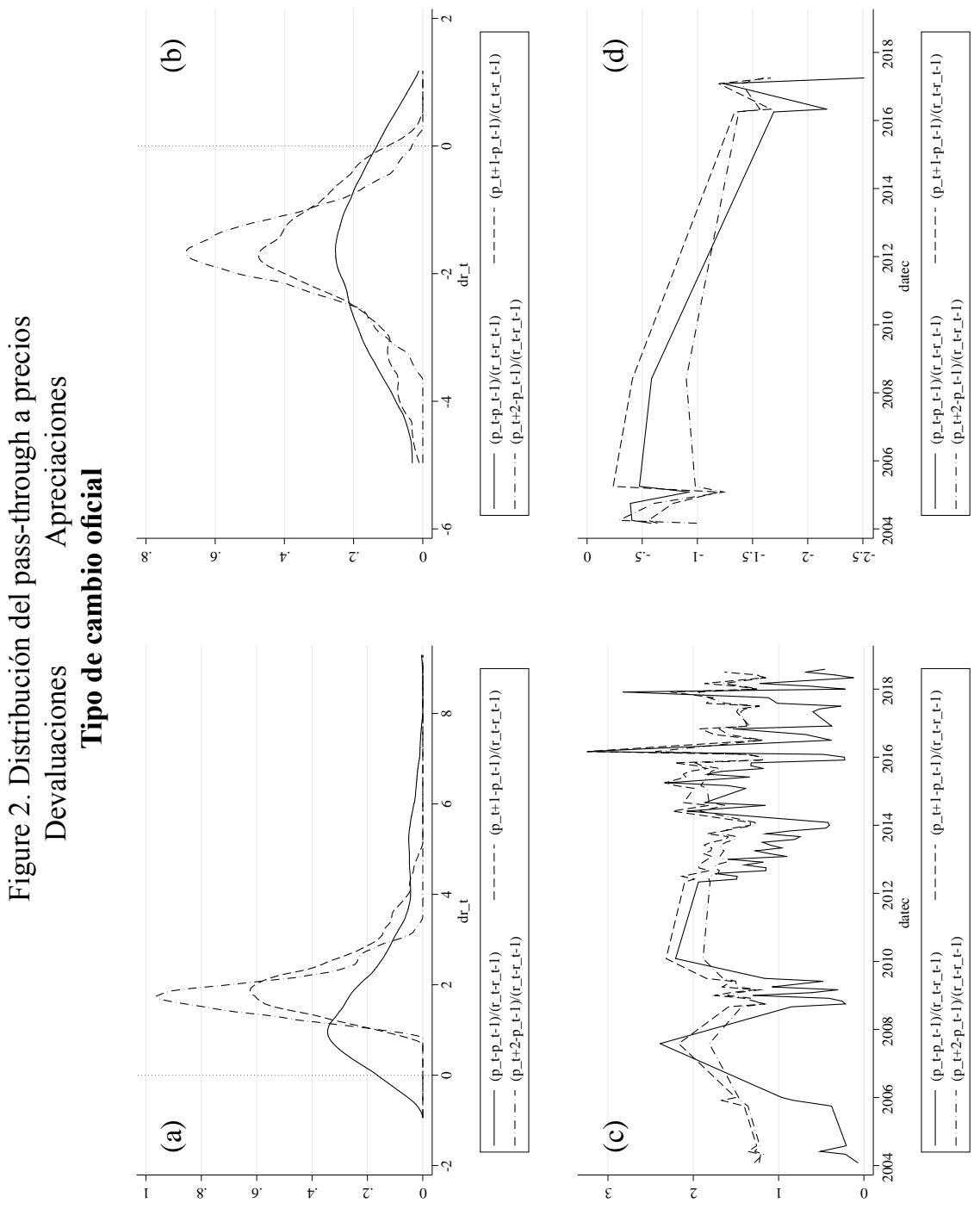

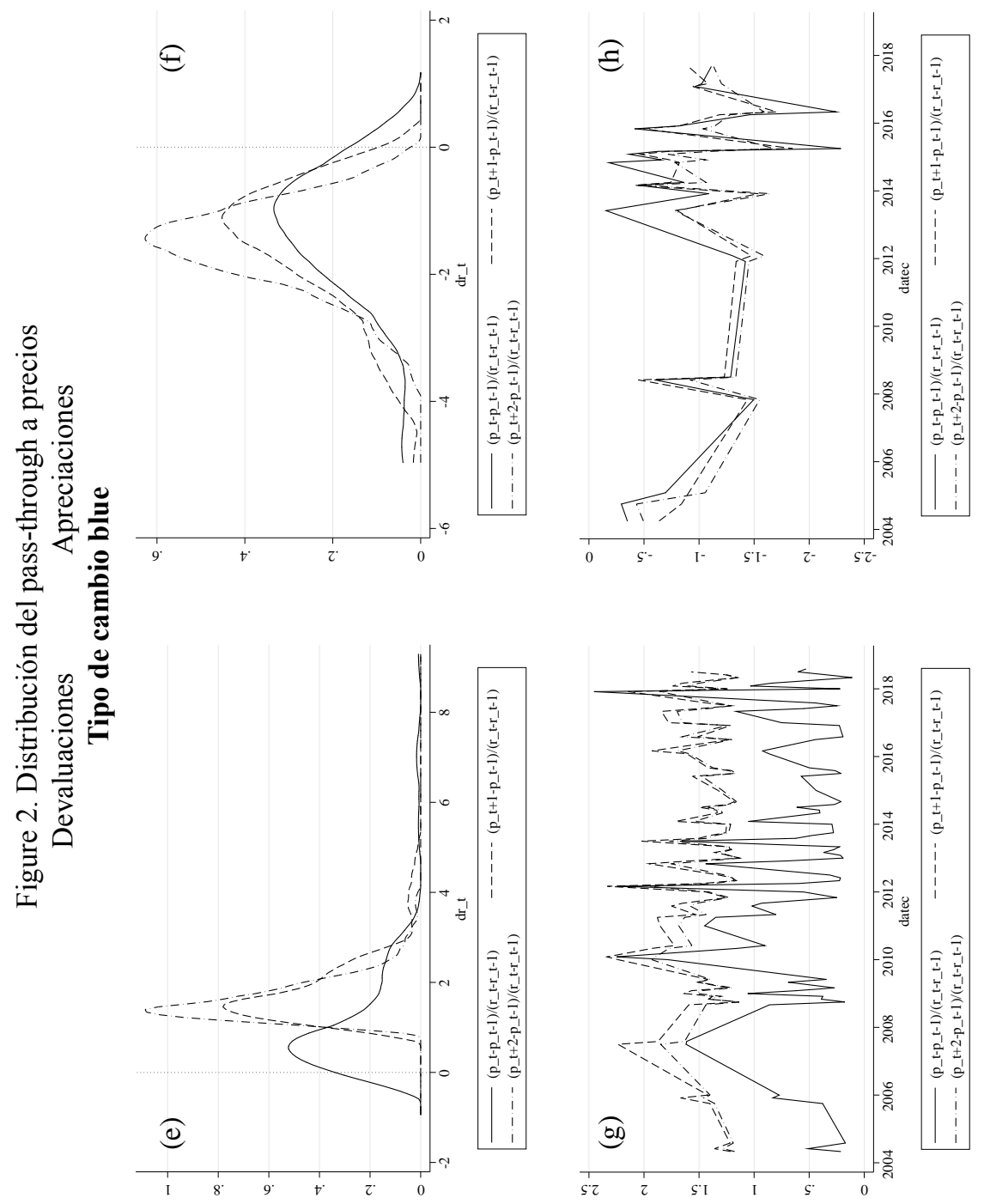

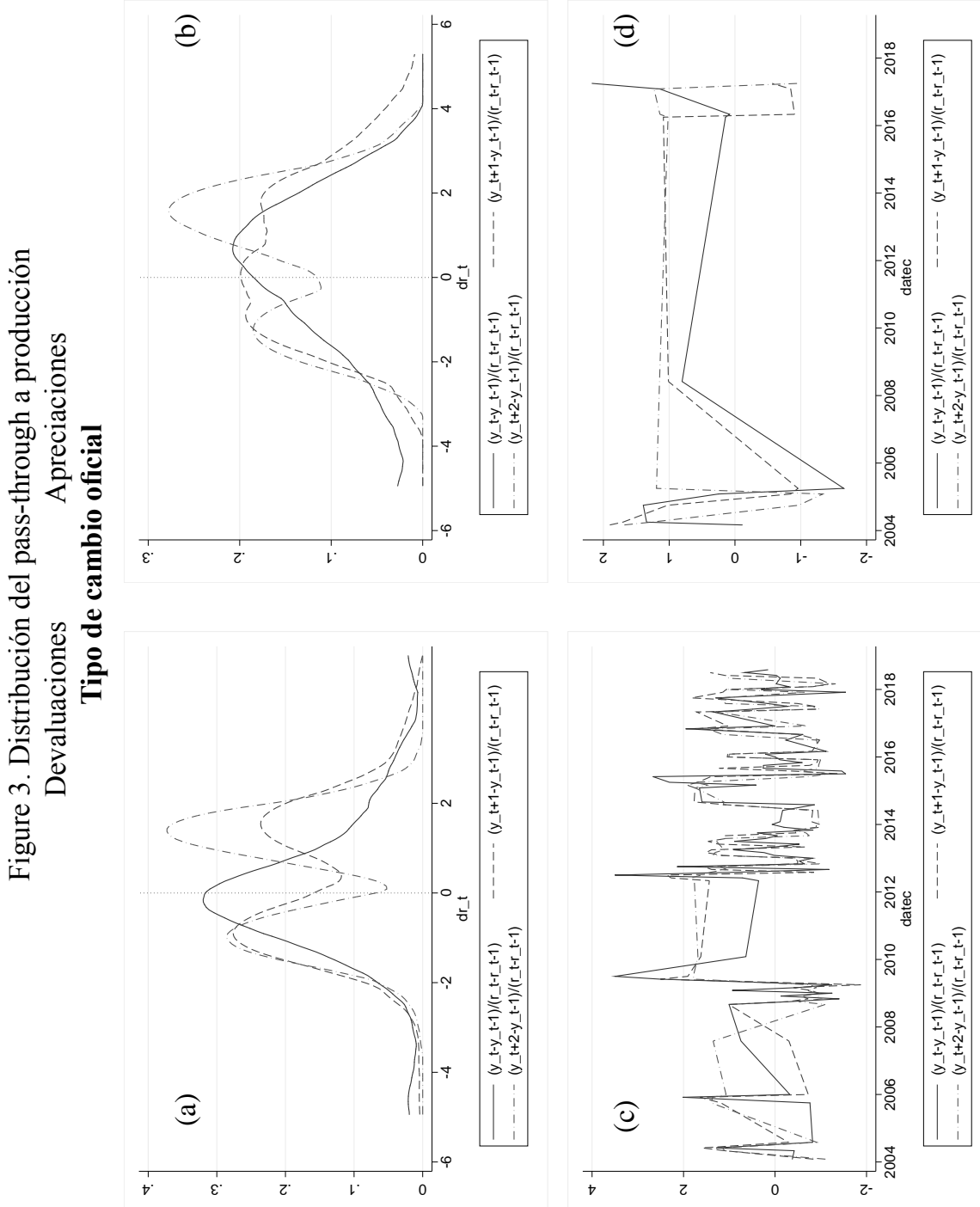

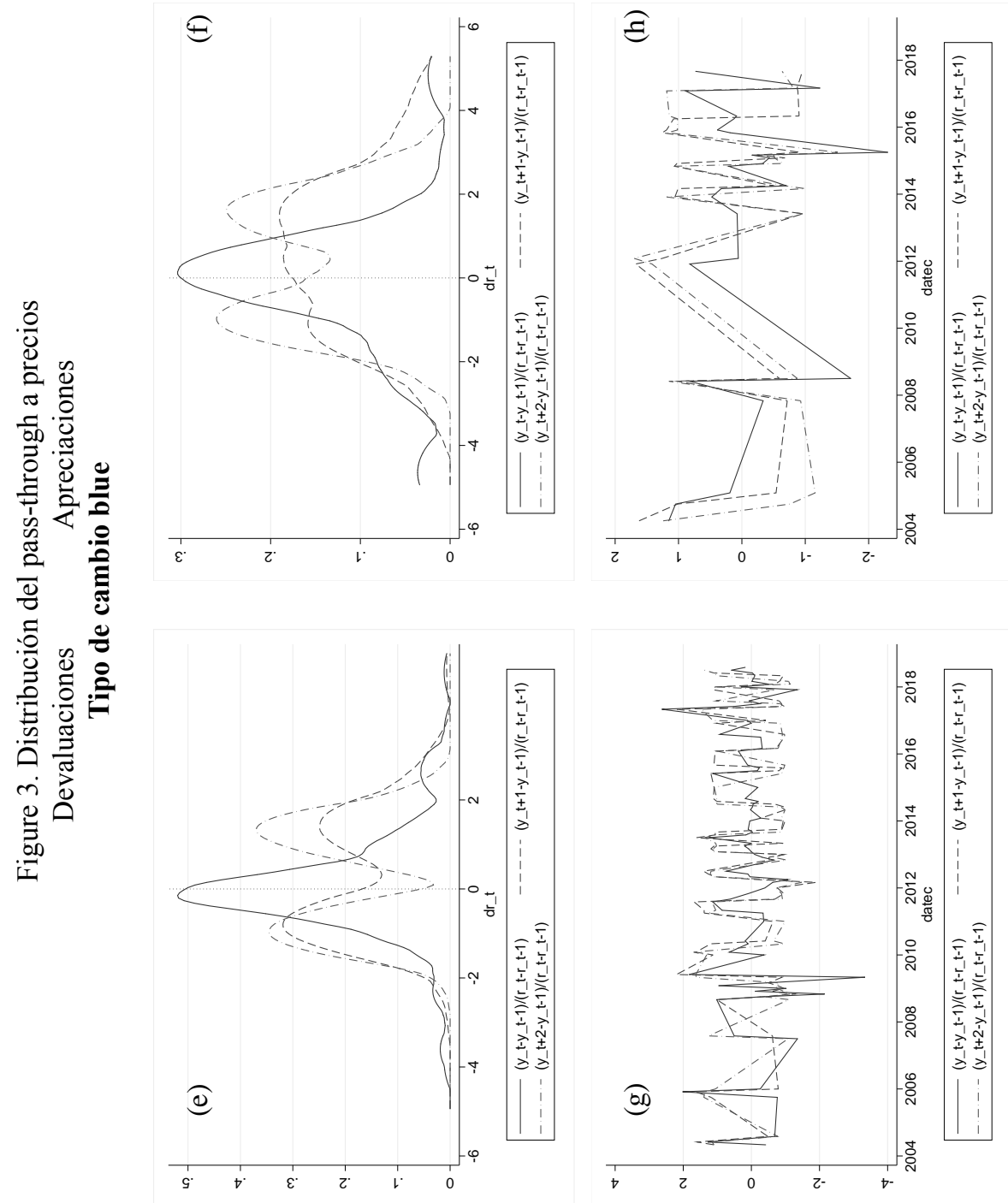
Figure 4. Series usadas para modelo econométrico

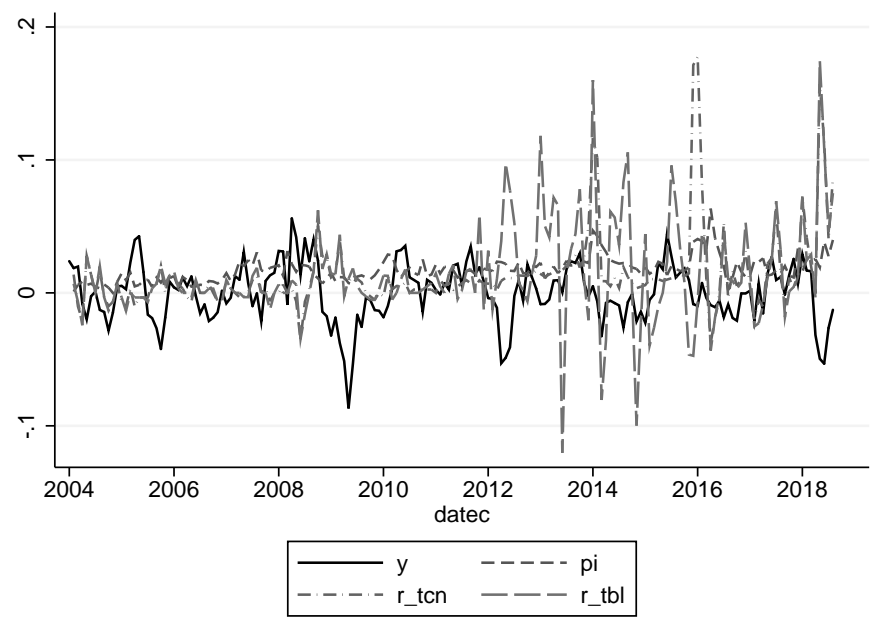

Nuestro objetivo es evaluar los efectos de un shock cambiario. Para identificar los shocks usamos una descomposición de Cholesky donde asumimos que $\rho_{\mathrm{t}}$ no tiene efectos contemporáneos en $\pi_{\mathrm{t}} \mathrm{e} \mathrm{y}_{\mathrm{t}}, \pi_{\mathrm{t}}$ afecta $\rho_{\mathrm{t}}$ pero no $\mathrm{y}_{\mathrm{t}}$, finalmente $\mathrm{y}_{\mathrm{t}}$ afecta $\rho_{\mathrm{t}} \mathrm{y} \pi_{\mathrm{t}}$. Este modelo implica que el tipo de cambio es una variable nominal que ajusta primero y que no afecta a las demás contemporáneas, lo cual es compatible con la inercia de las variables reales y costos de menú. Se estiman entonces los efectos de pass-through considerando un shock de variables están en diferencias. En particular, el shock se define como $\delta=(0,0,1)^{\prime}$. El análisis se realiza separadamente para el tipo de cambio oficial y el blue.

Primero, se computan los FIR a partir del análisis VAR tradicional, usando MCO como base para la estimación. Segundo, se utilizan los modelos FIRQ donde se varía un cuantil de cada ecuación mientras se mantienen los otros en la mediana. Consideremos los siguientes modelos FIRQ: (i) $\tau_{\mathrm{y}}=\{.10, .20 \ldots ., .90\}, \tau_{\pi}=\tau_{\rho}=.5$. $\tau_{\pi}=\{.10, .20 \ldots . .90\}, \tau_{\mathrm{y}}=\tau_{\rho}=.5 . \mathrm{y}$ (iii) $\tau_{\rho}=\{.10, .20 \ldots ., .90\}, \tau_{\mathrm{y}}=\tau_{\mathrm{\rho}}=.50$. El modelo (i) estudia los efectos para distintos senderos de producto, donde los cuantiles bajos se corresponden con un sendero de recesión condicional, es decir donde el producto está sistemáticamente por debajo de su tendencia, y los cuantiles altos corresponden a senderos de crecimiento. En este caso se fijan las otras dos variables, inflación y tipo de cambio en la mediana. El modelo (ii) analiza la heterogeneidad en términos 
de senderos de inflación, manteniendo producto y tipo de cambio en la mediana. Finalmente el modelo (iii) usa la heterogeneidad en el tipo de cambio, es decir, senderos de baja o alta devaluación/apreciación.

Para evaluar estos modelos se consideran los modelos de regresiones que hacen a las formas reducidas VAR y VARQ. La tabla 1 reporta los modelos de regresión para el tipo de cambio oficial, mientras que la tabla 2 lo mismo para el tipo de cambio blue. En este caso, se reporta el modelo de MCO (que es la base del VAR en la media), y los modelos de cuantiles para $.10, .25, .50, .75$ y .90 . Los resultados muestran que la dinámica del producto depende mayormente de sí mismo (con un coeficiente autorregresivo estadísticamente significativo arriba de .60), excepto para los cuantiles altos donde la inflación afecta positivamente y el tipo de cambio en forma negativa. La inflación, por su parte, depende de sí misma y del tipo de cambio (oficial, no hay relación para el blue). El tipo de cambio oficial no se ve afectado por producto e inflación, pero el tipo de cambio blue contiene un efecto positivo de la inflación en los cuantiles altos.

Tabla 1. Modelos VAR en forma reducida, MCO y regresiones por cuantiles. Tipo de cambio oficial

\begin{tabular}{|c|c|c|c|c|c|c|}
\hline \multirow[b]{2}{*}{ VARIABLES } & \multirow[b]{2}{*}{ MCO } & \multicolumn{5}{|c|}{ Cuantiles } \\
\hline & & .10 & .25 & .50 & .75 & .90 \\
\hline \multicolumn{7}{|c|}{ Variable dep.: $y_{t}$} \\
\hline \multirow[t]{2}{*}{$\mathrm{y}_{\mathrm{t}-1}$} & $.675^{* * *}$ & $.796^{* * *}$ & $.741^{* * *}$ & $.612^{* * *}$ & $.612^{* * *}$ & $.625^{* * *}$ \\
\hline & $(.0555)$ & $(.118)$ & $(.0692)$ & $(.0734)$ & $(.0831)$ & $(.0634)$ \\
\hline \multirow[t]{2}{*}{$\pi_{\mathrm{t}-1}$} & $.238^{*}$ & -.0815 & .153 & .00597 & .209 & $.584^{* * *}$ \\
\hline & (.139) & $(.297)$ & $(.174)$ & $(.185)$ & $(.209)$ & $(.159)$ \\
\hline \multirow[t]{2}{*}{$\rho_{\mathrm{t}-1}$} & $-.0775^{*}$ & -.0591 & -.0376 & -.0276 & $-.121^{*}$ & $-.218^{* * *}$ \\
\hline & $(.0446)$ & $(.0950)$ & $(.0557)$ & $(.0590)$ & $(.0668)$ & $(.0510)$ \\
\hline \multirow[t]{2}{*}{ Const. } & -.00332 & $-.0180^{* * *}$ & $-.0129^{* * *}$ & $-1.59 \mathrm{e}-05$ & $.00813^{* *}$ & $.0126^{* * *}$ \\
\hline & $(.00258)$ & $(.00549)$ & $(.00321)$ & $(.00341)$ & $(.00386)$ & $(.00294)$ \\
\hline $\mathrm{R} 2$ & .485 & & & & & \\
\hline
\end{tabular}


UNA EVALUACIÓN DEL PASS-THROUGH EN LA ARGENTINA USANDO FUNCIONES...

\begin{tabular}{|c|c|c|c|c|c|c|}
\hline \multicolumn{7}{|c|}{ Variable dep.: $\pi_{\mathrm{t}}$} \\
\hline$y_{t-1}$ & .0322 & .0319 & .0279 & $.0462^{* *}$ & .0290 & .00705 \\
\hline & $(.0227)$ & $(.0294)$ & $(.0250)$ & $(.0215)$ & $(.0288)$ & $(.0771)$ \\
\hline \multirow[t]{2}{*}{$\pi_{\mathrm{t}-1}$} & $.632^{* * *}$ & $.524^{* * *}$ & $.628^{* * *}$ & $.611^{* * *}$ & $.606^{* * *}$ & $.728^{* * *}$ \\
\hline & $(.0571)$ & $(.0738)$ & $(.0627)$ & $(.0541)$ & $(.0724)$ & (.194) \\
\hline \multirow[t]{2}{*}{$\rho_{\mathrm{t}-1}$} & $.0643^{* * *}$ & $.0702^{* * *}$ & $.0649^{* * *}$ & $.0704^{* * *}$ & $.0487^{* *}$ & .0926 \\
\hline & $(.0183)$ & $(.0236)$ & $(.0201)$ & $(.0173)$ & $(.0232)$ & $(.0620)$ \\
\hline \multirow[t]{2}{*}{ Const. } & $.00577^{* * *}$ & .000996 & $.00222^{*}$ & $.00539^{* * *}$ & $.00916^{* * *}$ & $.0104^{* * *}$ \\
\hline & $(.00105)$ & $(.00136)$ & $(.00116)$ & $(.00100)$ & $(.00134)$ & $(.00358)$ \\
\hline $\mathrm{R} 2$ & .521 & & & & & \\
\hline \multicolumn{7}{|c|}{ Variable dep.: $\rho_{\mathrm{t}}$} \\
\hline \multirow[t]{2}{*}{$\mathrm{y}_{\mathrm{t}-1}$} & -.0597 & -.104 & -.0110 & -.0207 & -.0173 & -.196 \\
\hline & $(.0847)$ & $(.0845)$ & $(.0537)$ & $(.0443)$ & $(.0651)$ & $(.249)$ \\
\hline \multirow[t]{2}{*}{$\pi_{\mathrm{t}-1}$} & .296 & -.0573 & .202 & $.236^{* *}$ & .248 & .691 \\
\hline & $(.213)$ & $(.212)$ & $(.135)$ & $(.111)$ & $(.163)$ & $(.627)$ \\
\hline \multirow[t]{2}{*}{$\rho_{\mathrm{t}-1}$} & $.551^{* * *}$ & $.246^{* * *}$ & $.315^{* * *}$ & $.519^{* * *}$ & $.672^{* * *}$ & $.816^{* * *}$ \\
\hline & $(.0681)$ & $(.0679)$ & $(.0432)$ & $(.0356)$ & $(.0523)$ & $(.201)$ \\
\hline \multirow[t]{2}{*}{ Const. } & .00111 & $-.00701^{*}$ & -.00408 & -.000873 & .00294 & .00937 \\
\hline & $(.00393)$ & $(.00392)$ & $(.00249)$ & $(.00206)$ & $(.00302)$ & $(.0116)$ \\
\hline $\mathrm{R} 2$ & .345 & & & & & \\
\hline
\end{tabular}

Notas: Errores estándar en paréntesis. ${ }^{*}$ significativo al $10 \%,{ }^{* *}$ significativo al $5 \%,{ }^{* * *}$ significativo al $1 \%$. 
Tabla 2: Modelos VAR en forma reducida, MCO y regresiones por cuantiles. Tipo de cambio blue

\begin{tabular}{|c|c|c|c|c|c|c|}
\hline \multirow[b]{2}{*}{ VARIABLES } & \multirow[b]{2}{*}{ MCO } & \multicolumn{5}{|c|}{ Cuantiles } \\
\hline & & .10 & .25 & .50 & .75 & .90 \\
\hline \multicolumn{7}{|c|}{ Variable dep.: $y_{t}$} \\
\hline \multirow[t]{2}{*}{$\mathrm{y}_{\mathrm{t}-1}$} & $.680^{* * *}$ & $.839^{* * *}$ & $.722^{* * *}$ & $.613^{* * *}$ & $.614^{* * *}$ & $.643^{* * *}$ \\
\hline & $(.0552)$ & $(.112)$ & $(.0711)$ & $(.0753)$ & $(.0853)$ & $(.0435)$ \\
\hline \multirow[t]{2}{*}{$\pi_{\mathrm{t}-1}$} & .176 & -.0339 & .121 & -.0152 & .165 & $.471^{* * *}$ \\
\hline & $(.131)$ & $(.266)$ & (.169) & $(.179)$ & $(.203)$ & $(.104)$ \\
\hline \multirow[t]{2}{*}{$\rho_{\mathrm{t}-1}$} & $-.0578^{*}$ & .0369 & -.0189 & -.0469 & -.0813 & $-.195^{* * *}$ \\
\hline & $(.0331)$ & $(.0671)$ & $(.0427)$ & $(.0452)$ & $(.0512)$ & $(.0261)$ \\
\hline \multirow[t]{2}{*}{ Const. } & -.00249 & $-.0197^{* * *}$ & $-.0126^{* * *}$ & .000532 & $.00883^{* *}$ & $.0140^{* * *}$ \\
\hline & $(.00257)$ & $(.00521)$ & $(.00331)$ & $(.00351)$ & $(.00398)$ & $(.00203)$ \\
\hline $\mathrm{R} 2$ & .485 & & & & & \\
\hline \multicolumn{7}{|c|}{ Variable dep.: $\pi_{t}$} \\
\hline \multirow[t]{2}{*}{$\mathrm{y}_{\mathrm{t}-1}$} & .0224 & .0296 & .0254 & .0266 & .0346 & -.0113 \\
\hline & $(.0234)$ & $(.0261)$ & $(.0247)$ & $(.0217)$ & $(.0262)$ & $(.100)$ \\
\hline \multirow[t]{2}{*}{$\pi_{\mathrm{t}-1}$} & $.699^{* * *}$ & $.580^{* * *}$ & $.635^{* * *}$ & $.703^{* * *}$ & $.670^{* * *}$ & $.817^{* * *}$ \\
\hline & $(.0556)$ & $(.0622)$ & $(.0589)$ & $(.0518)$ & $(.0624)$ & $(.239)$ \\
\hline \multirow[t]{2}{*}{$\rho_{\mathrm{t}-1}$} & .00897 & -.0128 & .0225 & .0159 & -.00794 & .00517 \\
\hline & $(.0140)$ & $(.0157)$ & $(.0148)$ & $(.0130)$ & $(.0157)$ & $(.0602)$ \\
\hline \multirow[t]{2}{*}{ Const. } & $.00533^{* * *}$ & .000740 & $.00204^{*}$ & $.00456^{* * *}$ & $.00876^{* * *}$ & $.00963^{* *}$ \\
\hline & $(.00109)$ & $(.00122)$ & $(.00115)$ & $(.00101)$ & $(.00122)$ & $(.00468)$ \\
\hline $\mathrm{R} 2$ & .487 & & & & & \\
\hline \multicolumn{7}{|c|}{ Variable dep.: $\rho_{t}$} \\
\hline \multirow[t]{2}{*}{$\mathrm{y}_{\mathrm{t}-1}$} & -.179 & -.138 & -.0476 & -.0893 & -.125 & -.495 \\
\hline & $(.122)$ & $(.281)$ & $(.103)$ & $(.0803)$ & $(.166)$ & $(.318)$ \\
\hline
\end{tabular}




\begin{tabular}{|c|c|c|c|c|c|c|}
\hline$\pi_{\mathrm{t}-1}$ & .313 & -.635 & -.0559 & .271 & $1.129^{* * *}$ & $2.030^{* * *}$ \\
\hline & $(.291)$ & $(.670)$ & $(.246)$ & $(.191)$ & $(.395)$ & $(.758)$ \\
\hline \multirow[t]{2}{*}{$\rho_{\mathrm{t}-1}$} & $.304^{* * *}$ & .137 & $.289^{* * *}$ & $.368^{* * *}$ & $.464^{* * *}$ & .298 \\
\hline & $(.0732)$ & $(.169)$ & (.0619) & $(.0482)$ & $(.0994)$ & $(.191)$ \\
\hline \multirow[t]{2}{*}{ Const. } & .00411 & -.00633 & -.00265 & $-6.45 e-06$ & .000841 & .0127 \\
\hline & $(.00569)$ & $(.0131)$ & $(.00481)$ & $(.00375)$ & $(.00772)$ & $(.0148)$ \\
\hline $\mathrm{R} 2$ & .119 & & & & & \\
\hline
\end{tabular}

Notas: Errores estándar en paréntesis. ${ }^{*}$ significativo al $10 \%,{ }^{* *}$ significativo al $5 \%,{ }^{* * *}$ significativo al $1 \%$.

Sobre la base de las regresiones de cuantiles se construyen las FIR. Consideremos primero los efectos de un shock del tipo de cambio (aumento de la tasa de devaluación) sobre las variables del sistema en términos del modelo VAR tradicional, es decir, basados en la media de las variables, esto es, usando VAR-FIR tradicionales. El efecto de largo plazo sobre el producto es negativo con una elasticidad cercana a 0.4 para el tipo de cambio oficial y arriba de 0.2 para el tipo de cambio blue. En términos de la inflación, el efecto de largo plazo es positivo alrededor de 0.4 para el tipo de cambio oficial, pero el efecto aparece como cercano a 0 cuando se evalúa el tipo de cambio blue. El último resultado puede resultar extraño. Sin embargo, el mismo se debe al hecho de que estamos considerando un modelo en diferencias. Así, un shock en $\rho_{\mathrm{t}}$ usando uno u otro tipo de cambio puede significar cosas diferentes. Si usamos el tipo de cambio oficial, un shock puede ser el resultado de un atraso (apreciación) insostenible del tipo de cambio, que deriva en un reajuste de precios de forma brusca cuando se devalúa. Si por otro lado usamos el tipo de cambio blue, los atrasos cambiarios ya hubieran sido absorbidos por el mercado paralelo, y por ende, un shock puede significar un reajuste de precios relativos. De hecho, la figura 1 muestra grandes diferencias entre ambos tipos de cambio para el período 2011-2015.

Existen, sin embargo, cuestiones metodológicas que hacen que se deba dudar de la representatividad de los modelos para la media. Al modelizar la media, los resultados se ven afectados por la asimetría en las respuestas. Es el punto central de este trabajo, que una medida alternativa de medición, que es en este sentido preferible, es la estimación de FIRQ. 

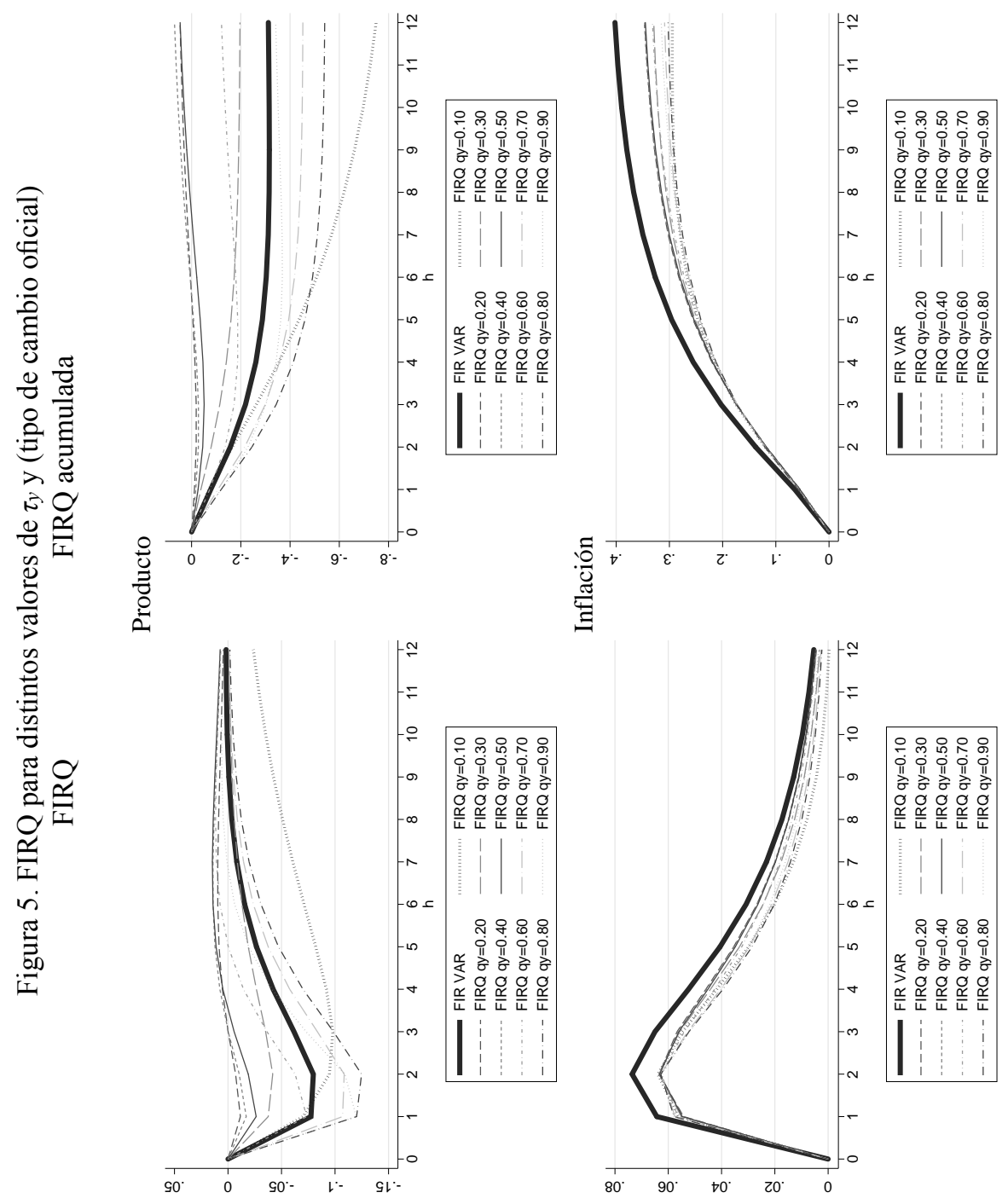


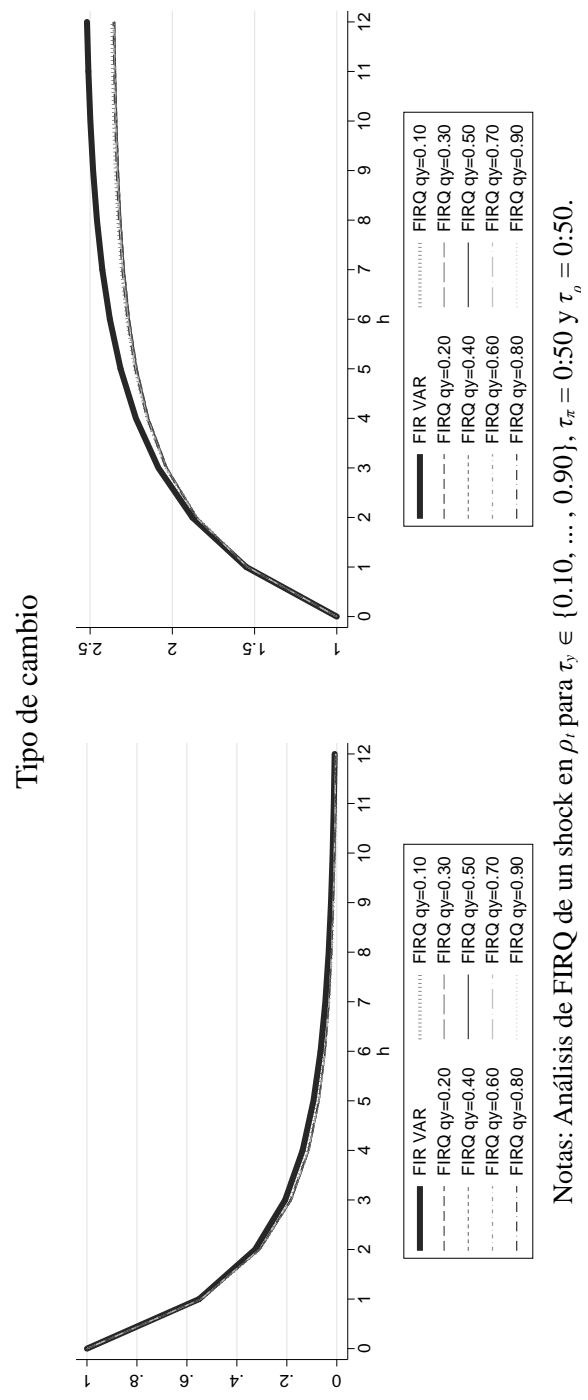



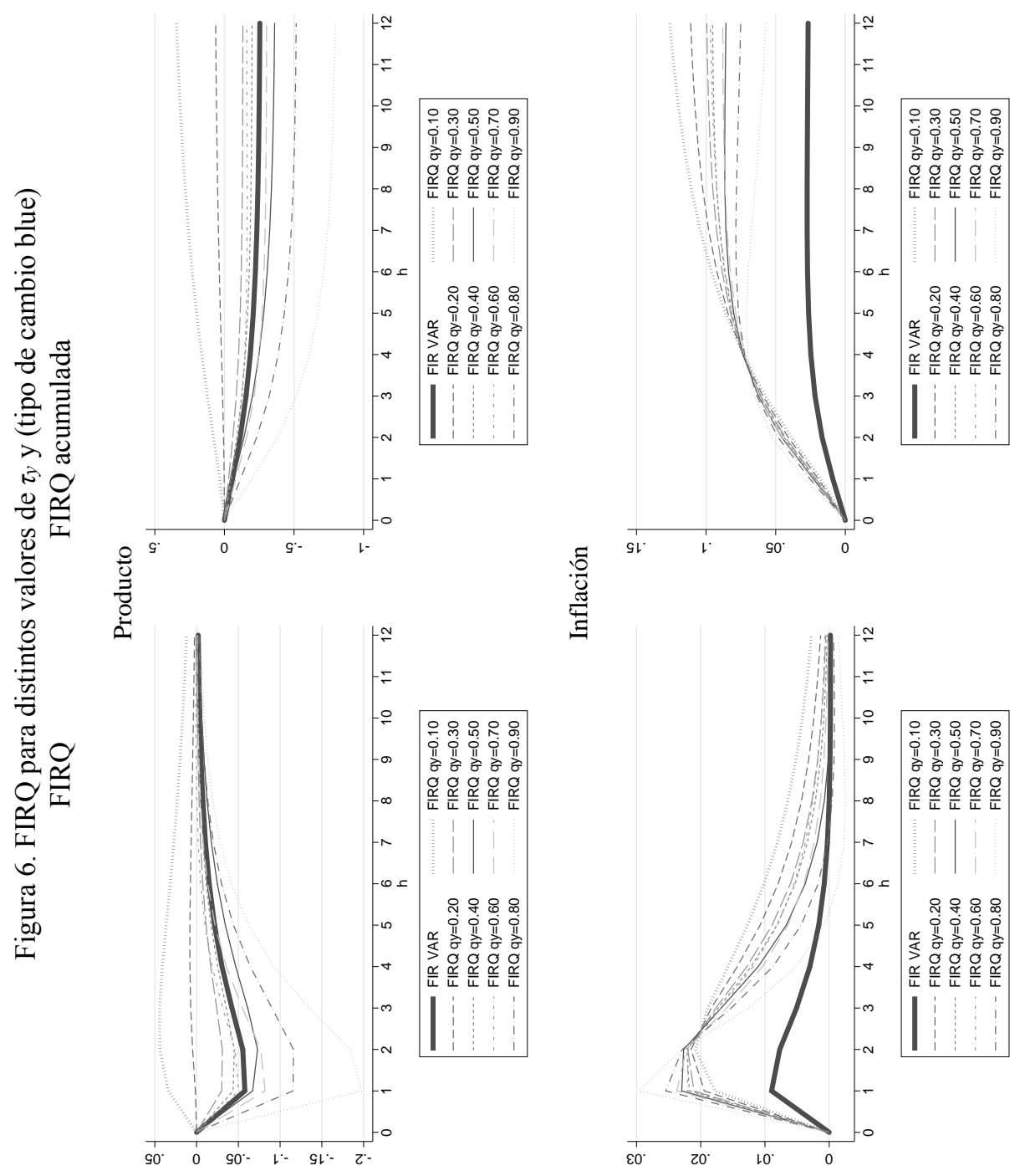


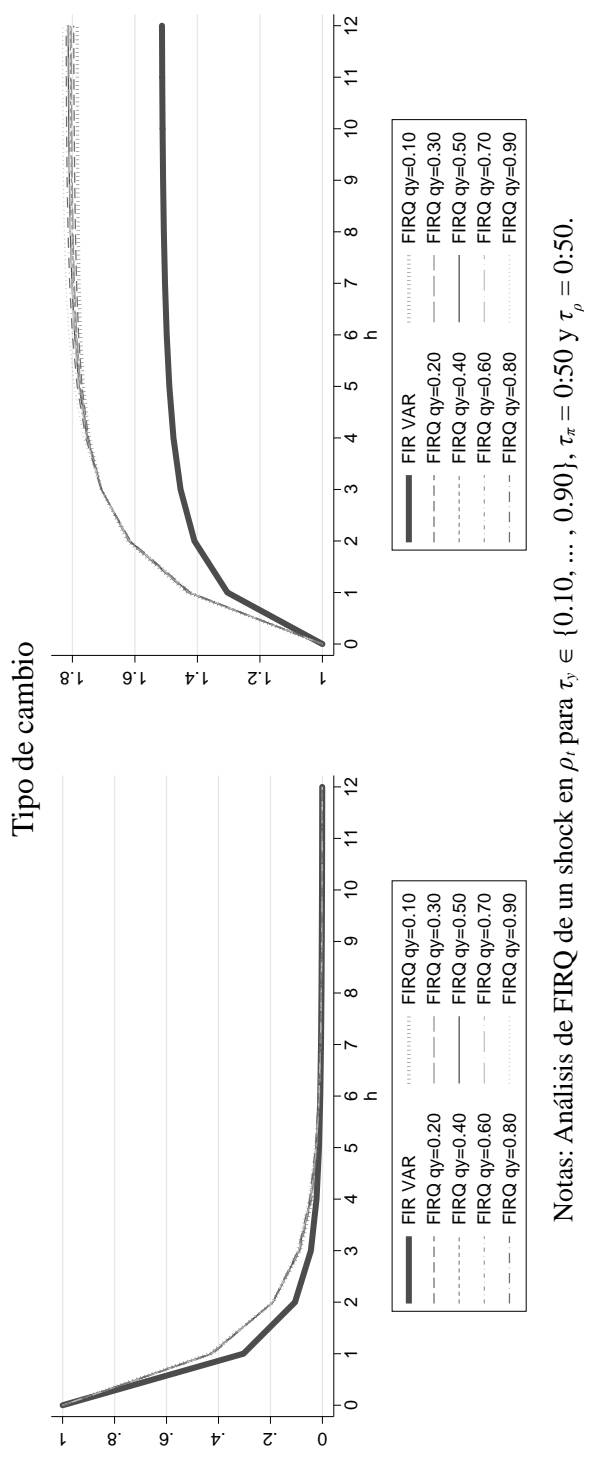


Consideremos primero los efectos de una devaluación sobre las variables del sistema evaluando la heterogeneidad en $\tau_{\mathrm{y}}$, es decir el modelo (i) en párrafos anteriores. Las figuras 5 y 6 reportan las FIRQ para el tipo de cambio oficial y blue, respectivamente, para variaciones en los cuantiles del producto. El tipo de cambio tiene un gran impacto sobre el producto: los efectos de largo plazo varían desde 0.8 a 0.1 para el tipo de cambio oficial, de 0.8 a 0.4 para el blue. De hecho la media se corresponde a una FIRQ con $\tau_{\mathrm{y}}=.9$, es decir, está influenciada por respuestas comparativamente altas del producto. Los mayores efectos se observan sin embargo para $\tau_{\mathrm{y}}=.1$ (con tipo de cambio oficial), es decir para una posición de recesión extrema, mientras que desciende bruscamente a 0 para $\tau_{\mathrm{y}}=.2$. Como resultado, los efectos de shocks en el tipo de cambio son más persistentes y negativos para las colas de la distribución condicional del producto, es decir, períodos de alto crecimiento o recesión. Esta heterogeneidad explica en parte los efectos no condicionales encontrados en las figuras 2 y 3 , donde se observaba una clara bimodalidad en los efectos. Es decir, se espera que los mayores efectos negativos sobre el producto se correspondan con situaciones donde se observa una posición extrema en el ciclo económico (desestacionalizado) tanto alto como bajo.

Teniendo en cuenta el pass-through a precios, podemos observar que el efecto de la FIR sobre la media no está contenido en los FIRQ que se analizan. En general se observa que el efecto de pass-through a largo plazo es mayor (menor) para cuantiles bajos (altos) del producto (en particular usando el tipo de cambio blue). De esta manera, habría un mayor traspaso a precios en períodos de recesión que de expansión.

Consideremos ahora la heterogeneidad en términos de los cuantiles de la inflación, $\tau_{\pi}$, modelo (ii). Las figuras 7 y 8 reportan las FIRQ para el tipo de cambio oficial y blue, respectivamente, para variaciones en $\tau_{\pi}$. En este caso, la mayor heterogeneidad se observa en la inflación. Cuando nos concentramos en el tipo de cambio oficial vemos que los efectos de pass-through a precios van de 0.15 a 0.45 en forma inversamente monótona con respecto a los cuantiles. Para cuantiles bajos de la inflación, los efectos de largo plazo están por encima de la media, y los menores efectos se observan para cuantiles altos. Lo anterior no aplica, sin embargo, para $\tau_{\pi}=.9$, que está en realidad por encima de la media. En resumen, esto indica que los efectos de un shock devaluatorio son mayores en contextos de baja inflación que en contextos de alta inflación (excepto extrema). Esto contradice los resultados propuestos en la literatura de Taylor (2000) y Edwards (2006) en cuanto a la relación de la magnitud del pass-through a precios y los niveles de inflación. 
Por otro lado, no hay un efecto significativo en cuanto a los efectos sobre el producto. Todos los FIRQ de cuantiles de inflación están cercanos a 0 y difieren de los efectos negativos de la media. De hecho, la dinámica de las FIRQ muestra que el producto cae durante los primeros seis meses, para luego recuperarse, con un efecto levemente positivo en el largo plazo.

Considerando ahora el tipo de cambio blue, los resultados son muy diferentes a los observados para el oficial. En términos de la inflación, la monotonicidad se pierde, sin un claro patrón de comportamiento. Para el producto, los efectos negativos de largo plazo siguen a los de la FIR en la media, con un valor más negativo aún.

Finalmente, utilizamos la heterogeneidad en los cuantiles condicionales del tipo de cambio, $\tau_{\rho}$, modelo (iii), en las figuras 9 y 10 para el oficial y el blue, respectivamente. Usando el tipo de cambio oficial se observan grandes cambios en los efectos. Cuando vemos el efecto sobre el producto se observa que los cuantiles altos del tipo de cambio (en diferencias) son compatibles con un efecto negativo de largo plazo sobre el producto. De hecho el efecto a 12 meses de $\tau_{\rho}=.9$ es similar al efecto de la media. De esa manera se puede entender en parte por qué el efecto sobre el producto usando VAR en la media es negativo. Ese proceso en particular está mayormente influenciado por un proceso de persistencia muy alto del tipo de cambio, es decir, una serie de devaluaciones por arriba del comportamiento histórico. Por otro lado, el pass-through a precios también se ve influenciado al variar $\tau_{\rho}$. Los efectos de largo plazo sobre la inflación son mayores a .6 para $\tau_{\rho} \geq .8, \mathrm{y}$ mayores a .4 para $\tau_{\rho} \geq .7$.

Para entender los resultados anteriores conviene evaluar los efectos autorregresivos del tipo de cambio. Si el efecto sobre la media de $\rho$ se corresponde con un efecto de magnitud 2, que implica que un shock de 1 unidad se duplica en promedio, se observa gran heterogeneidad en cuanto a la variabilidad a través de $\tau_{\rho}$.

Considerando el tipo de cambio blue, tenemos resultados en la misma dirección a lo observado con el tipo de cambio oficial. En este caso se observa menor heterogeneidad y menor amplitud en los efectos que con el tipo de cambio oficial.

Finalmente, a partir de los distintos senderos de cuantiles se estudia la distribución de la FIRQ para todos los modelos potenciales. Esto se realiza evaluando 1000 senderos de cuantiles aleatorios, es decir, simulando 1000 veces 3 variables uniformes independientes entre sí, cada uno correspondiente a un horizonte 

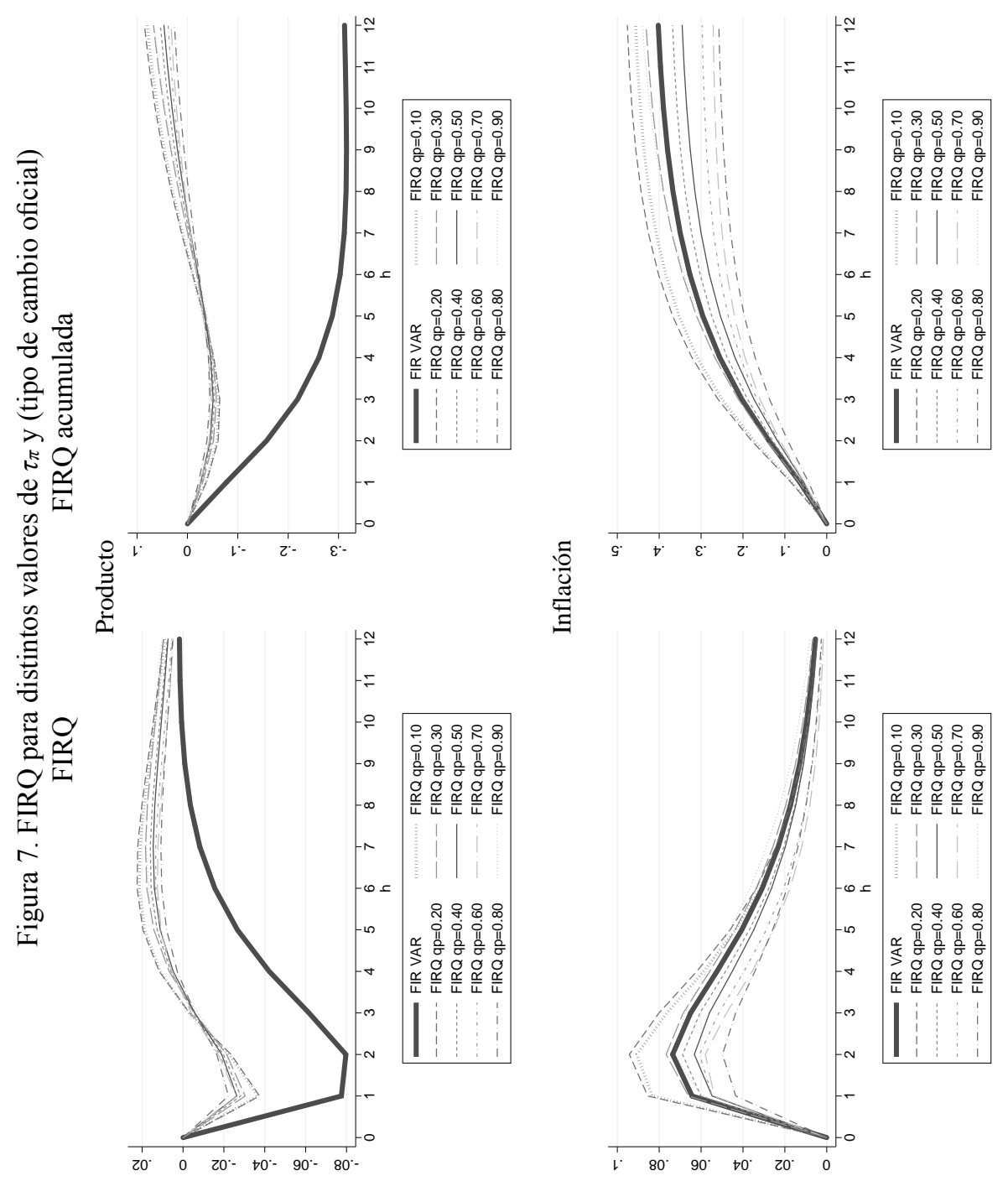


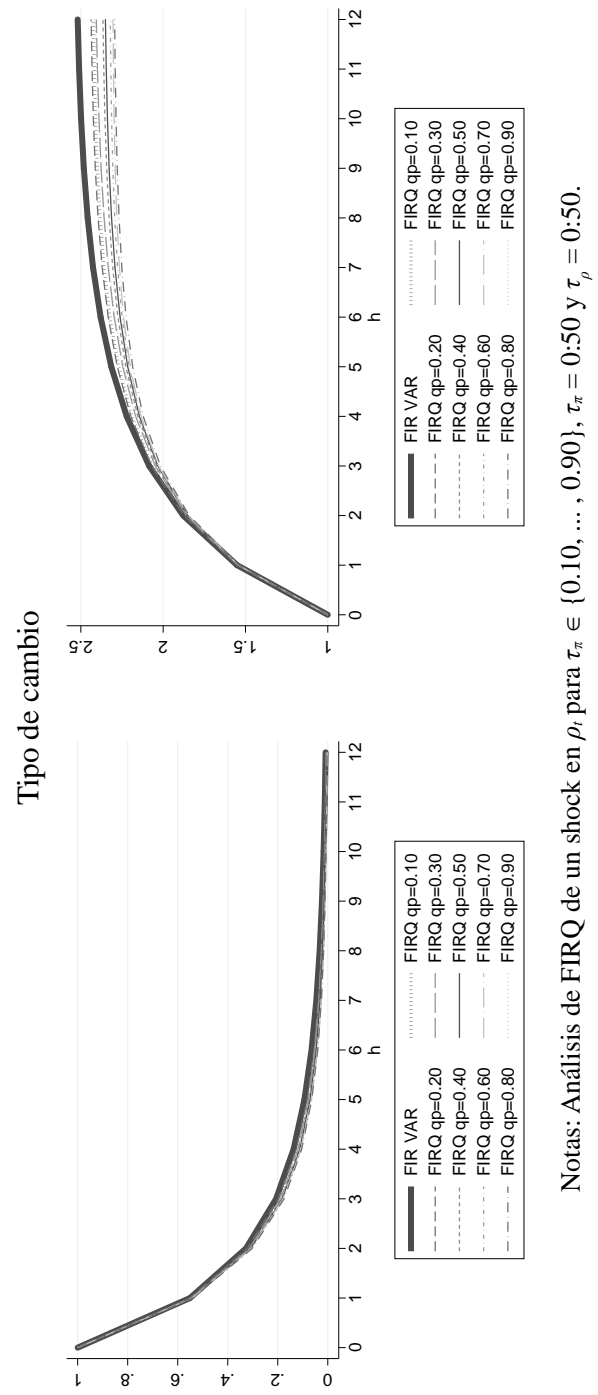




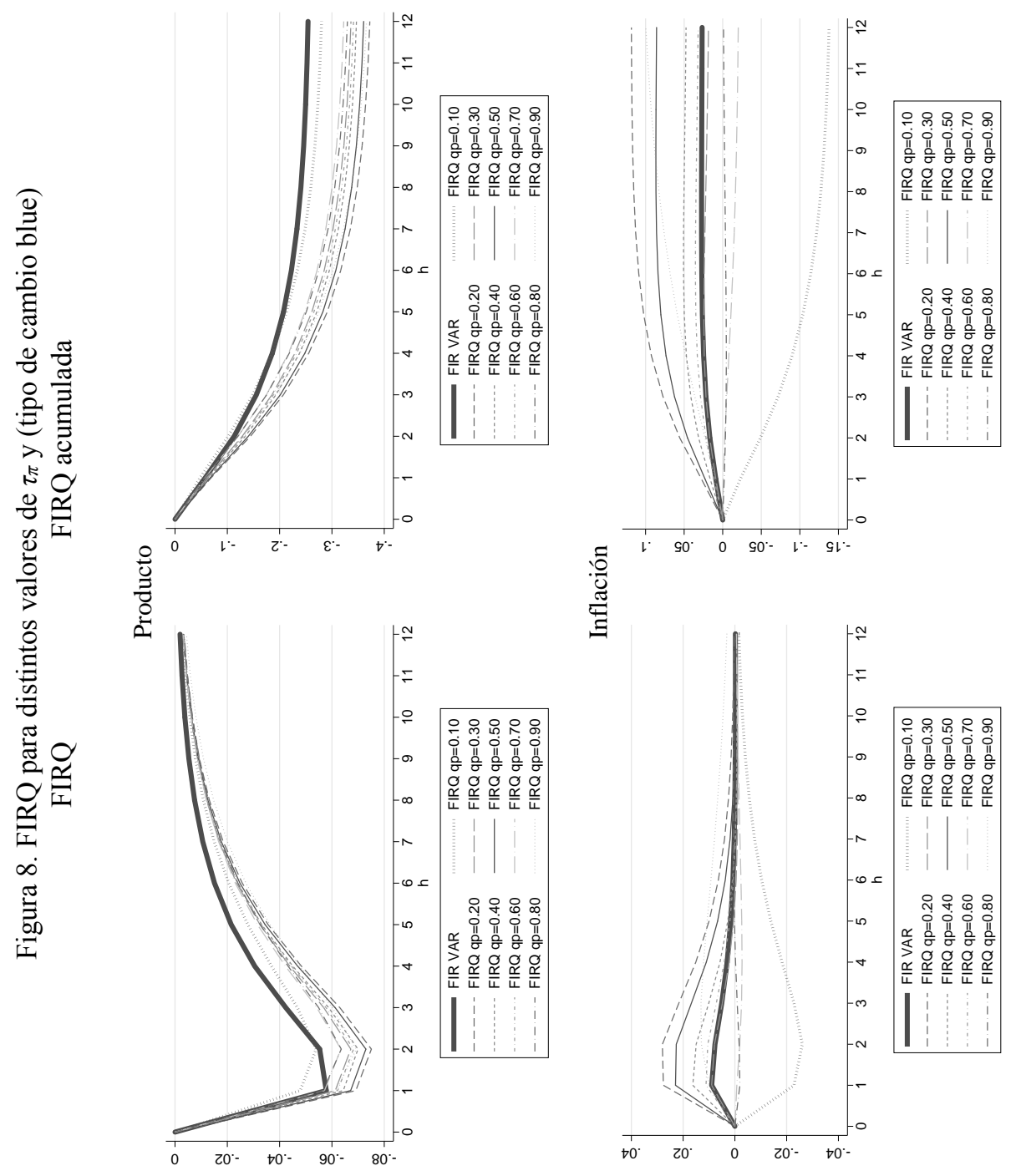




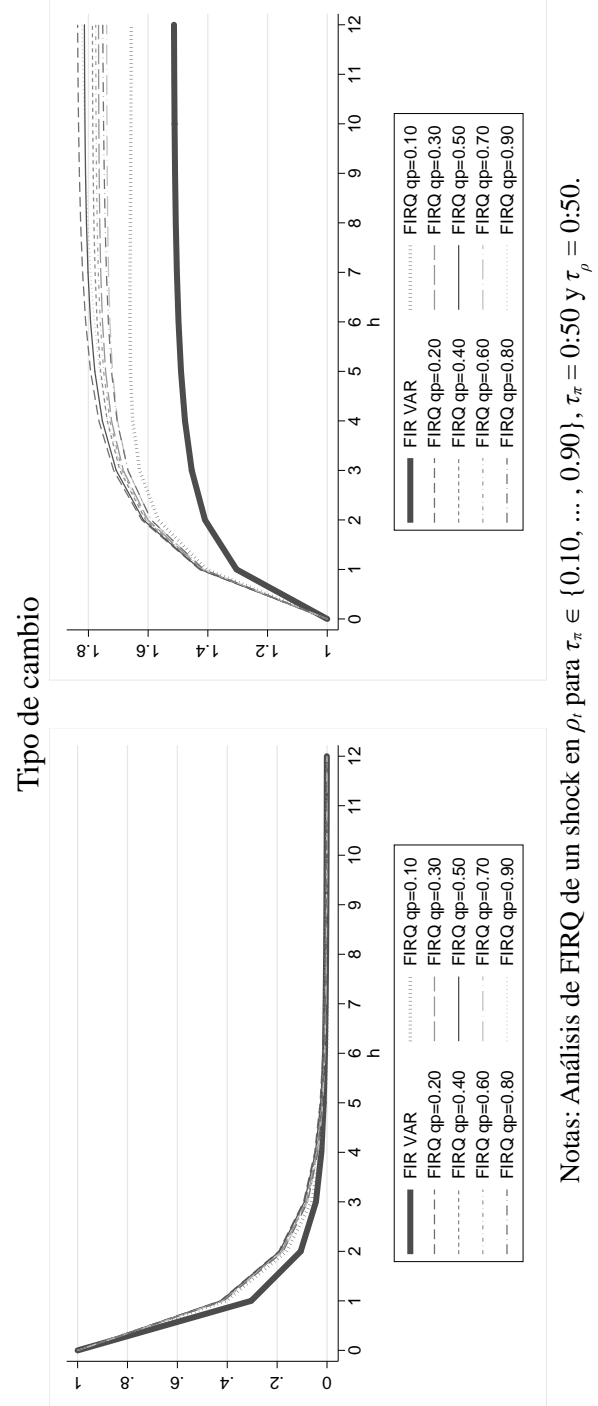




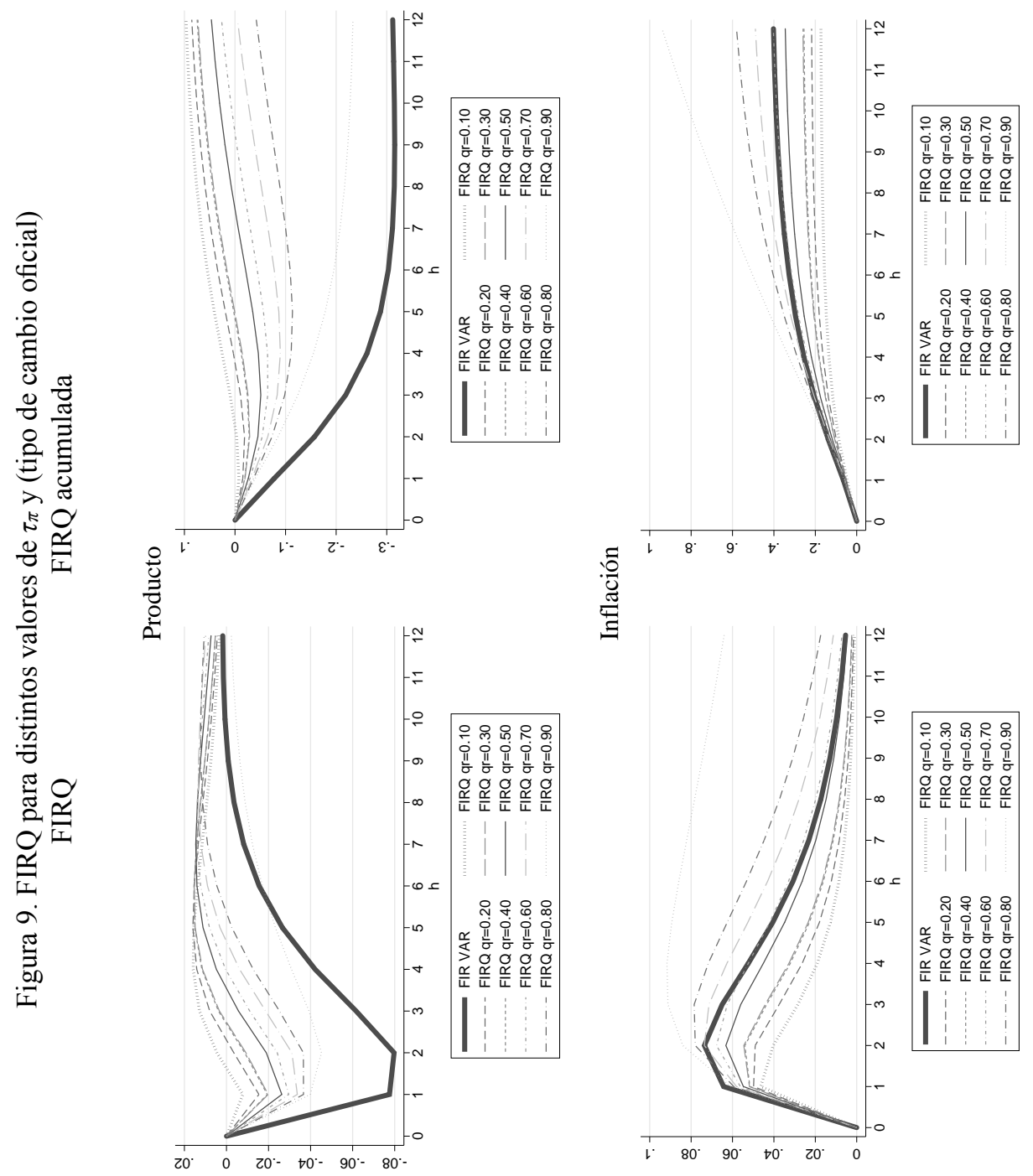




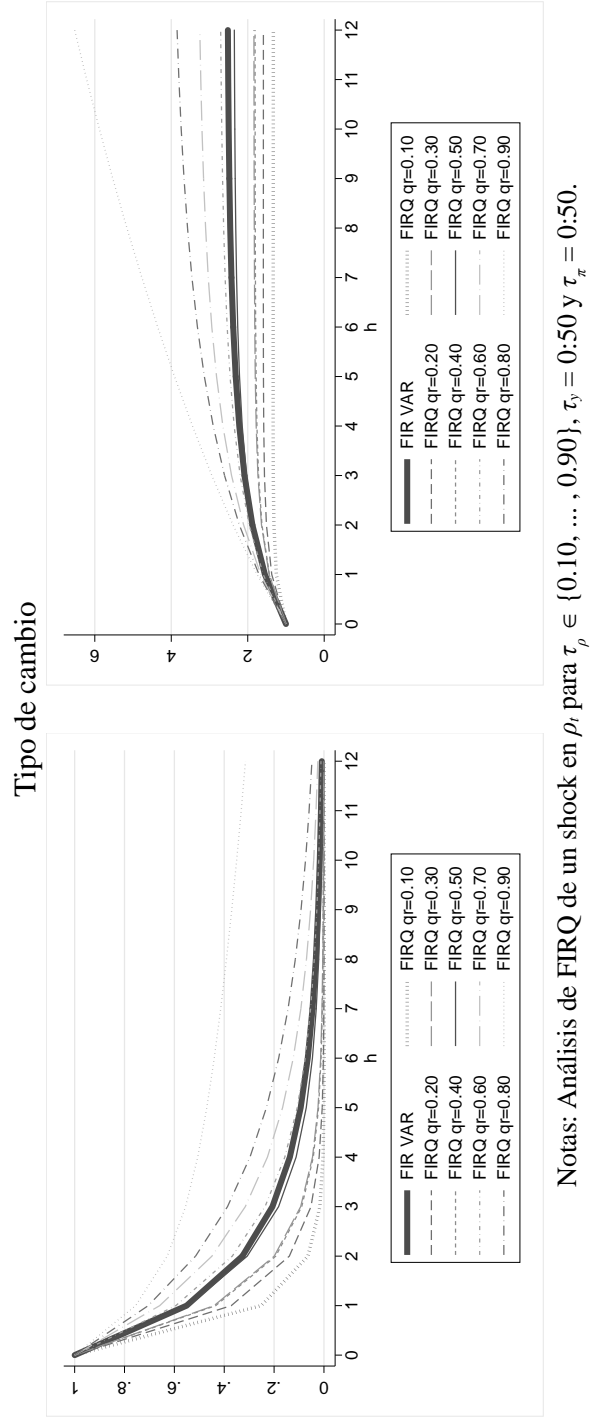




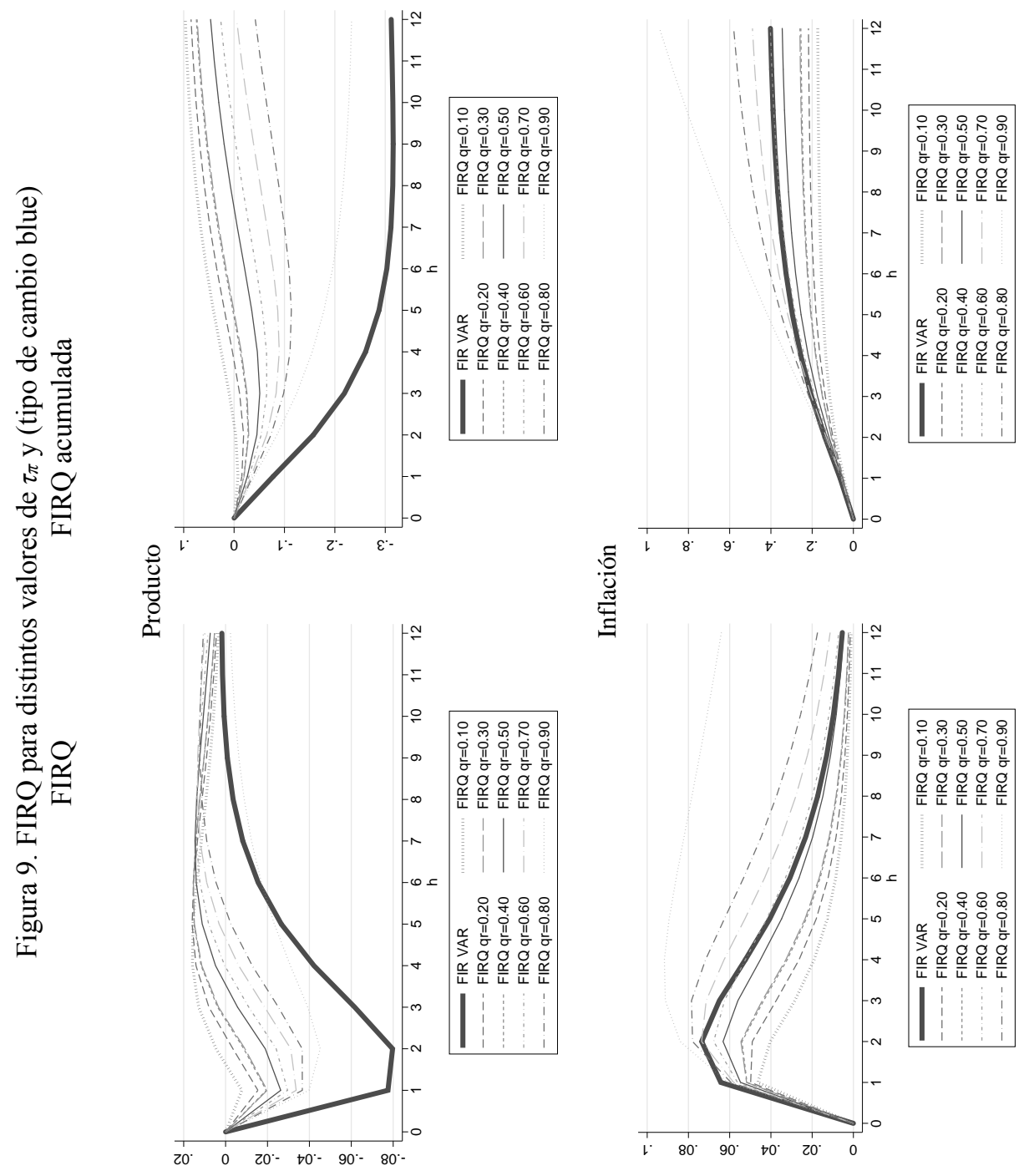


UNA EVALUACIÓN DEL PASS-THROUGH EN LA ARGENTINA USANDO FUNCIONES...

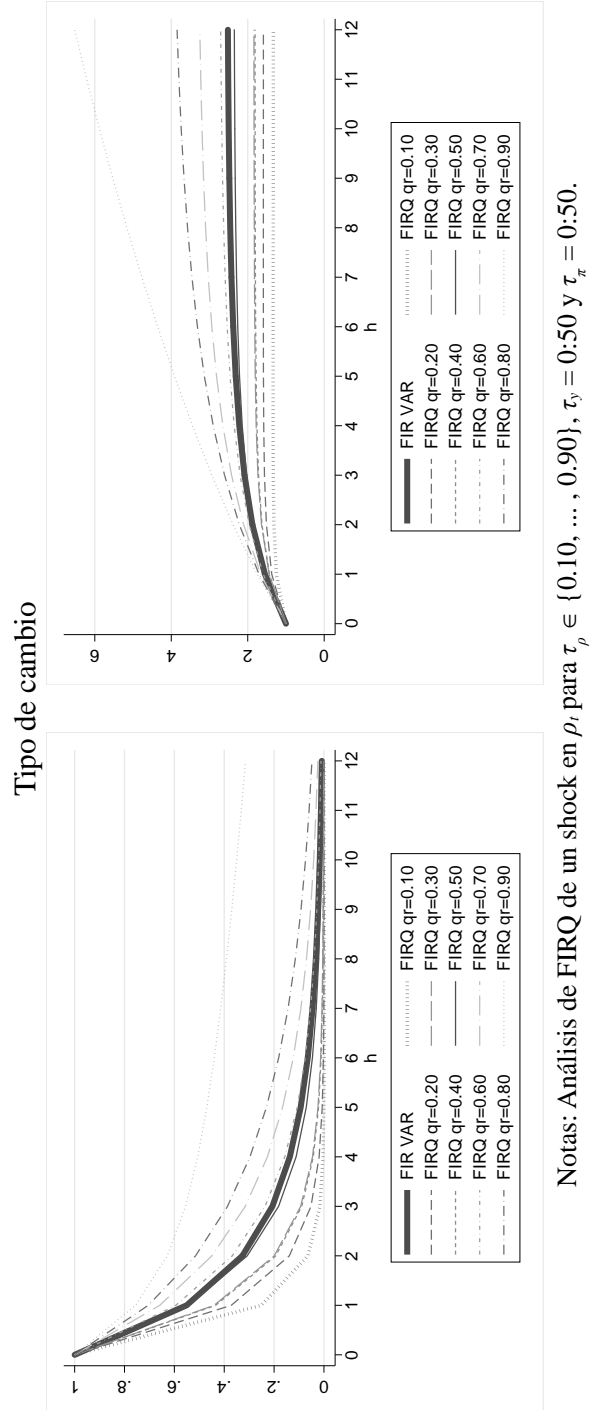




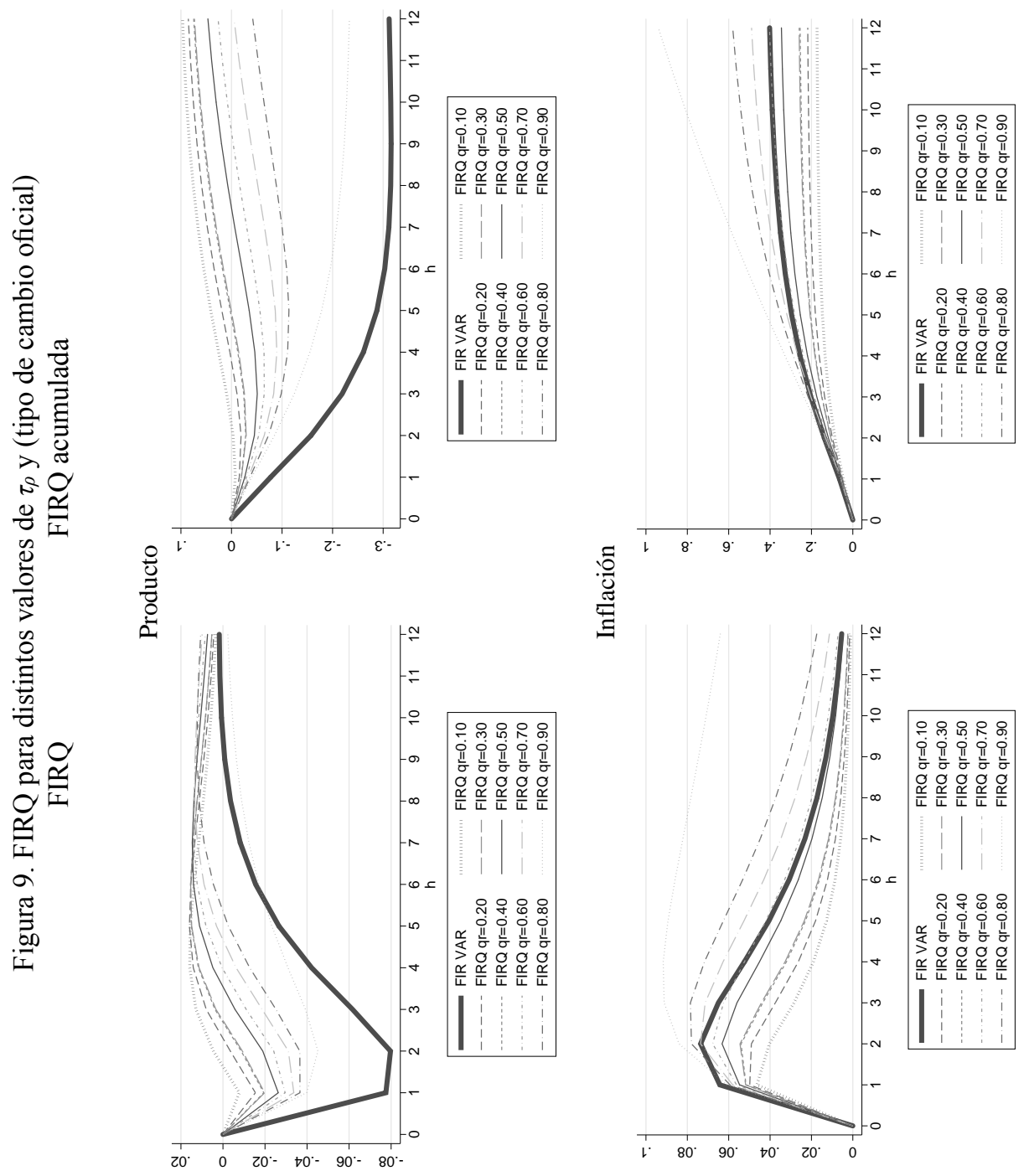




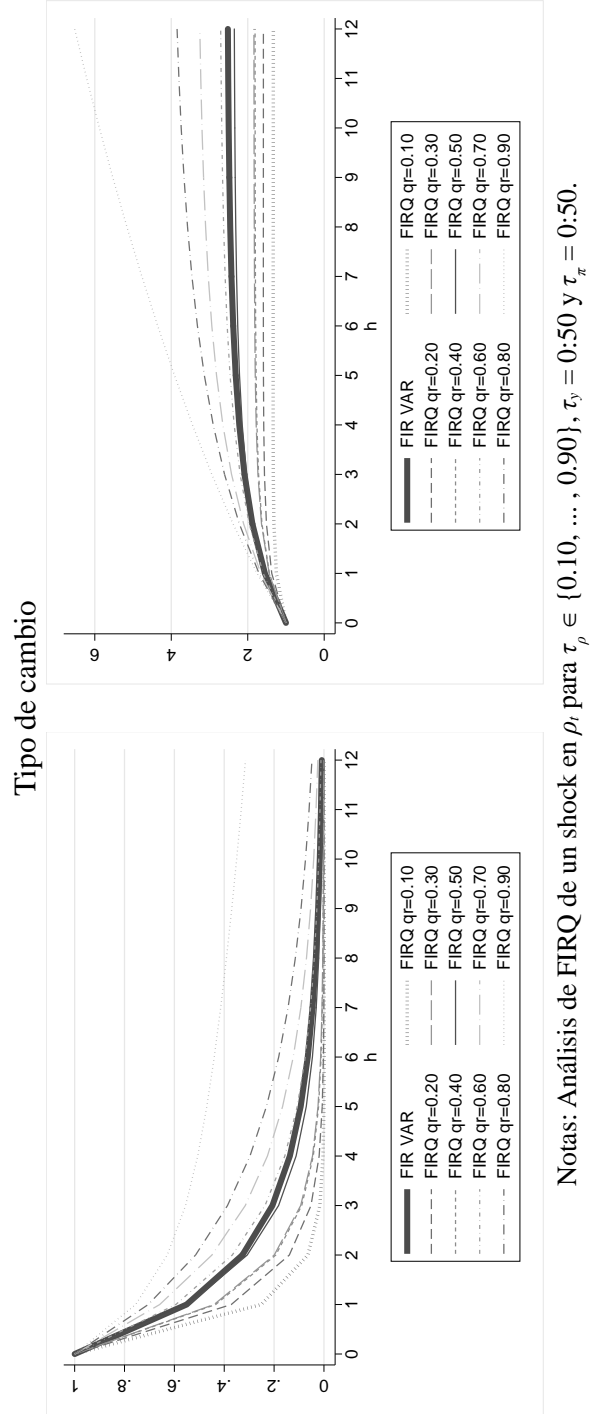



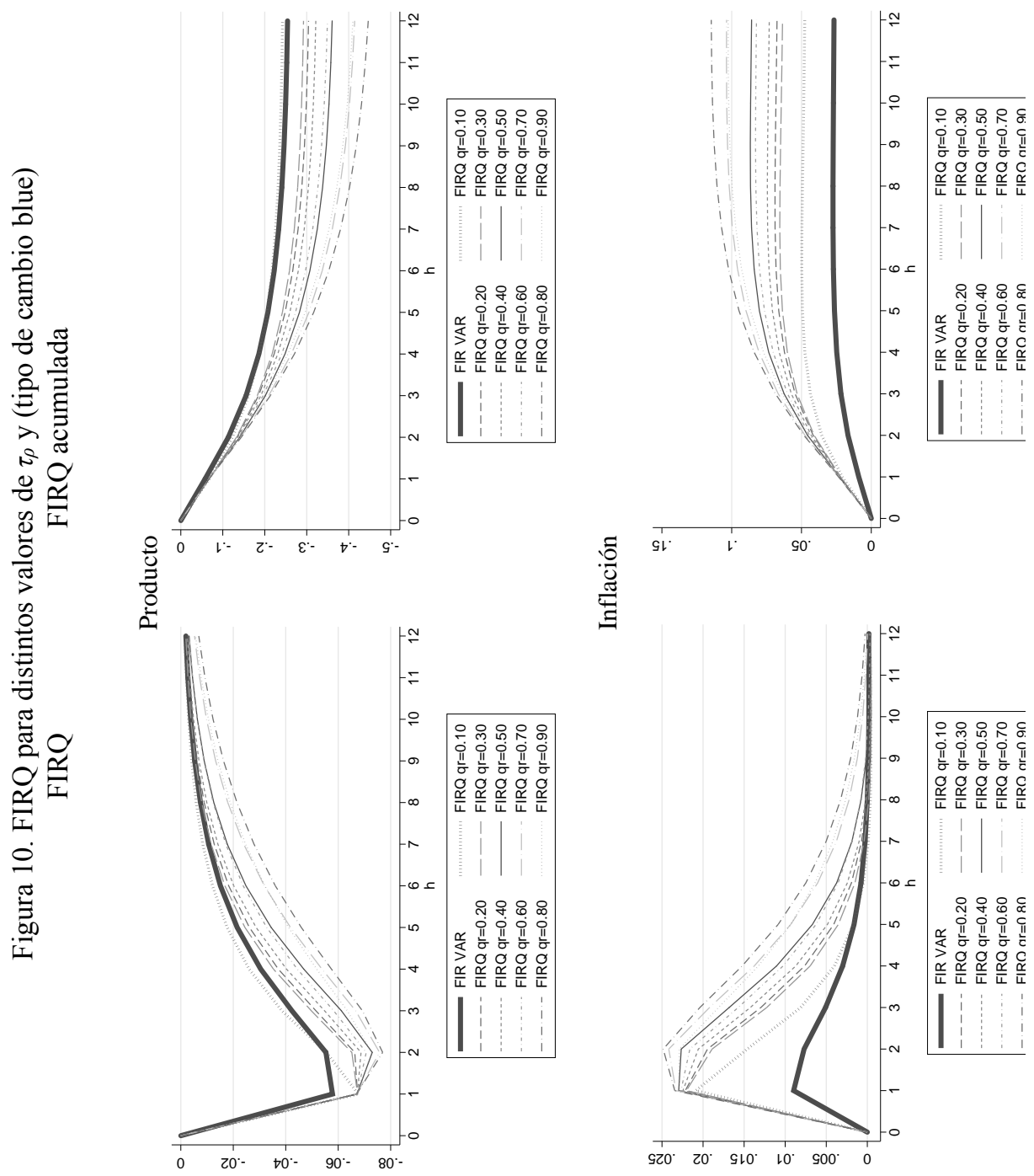


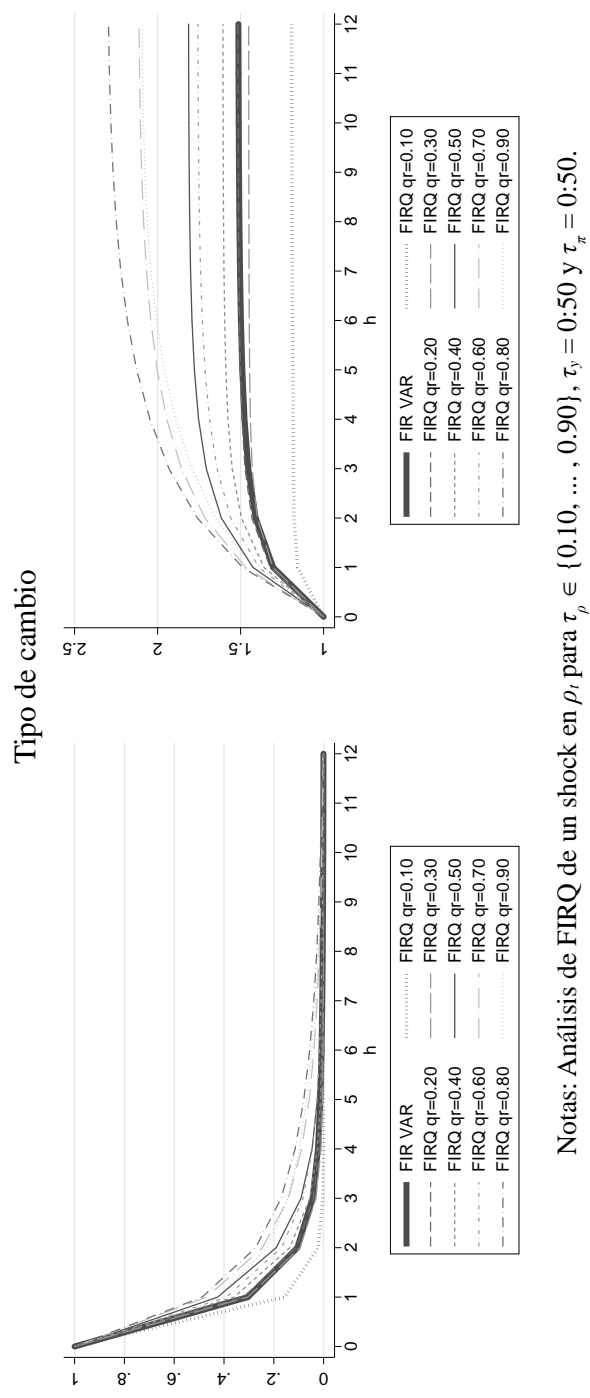




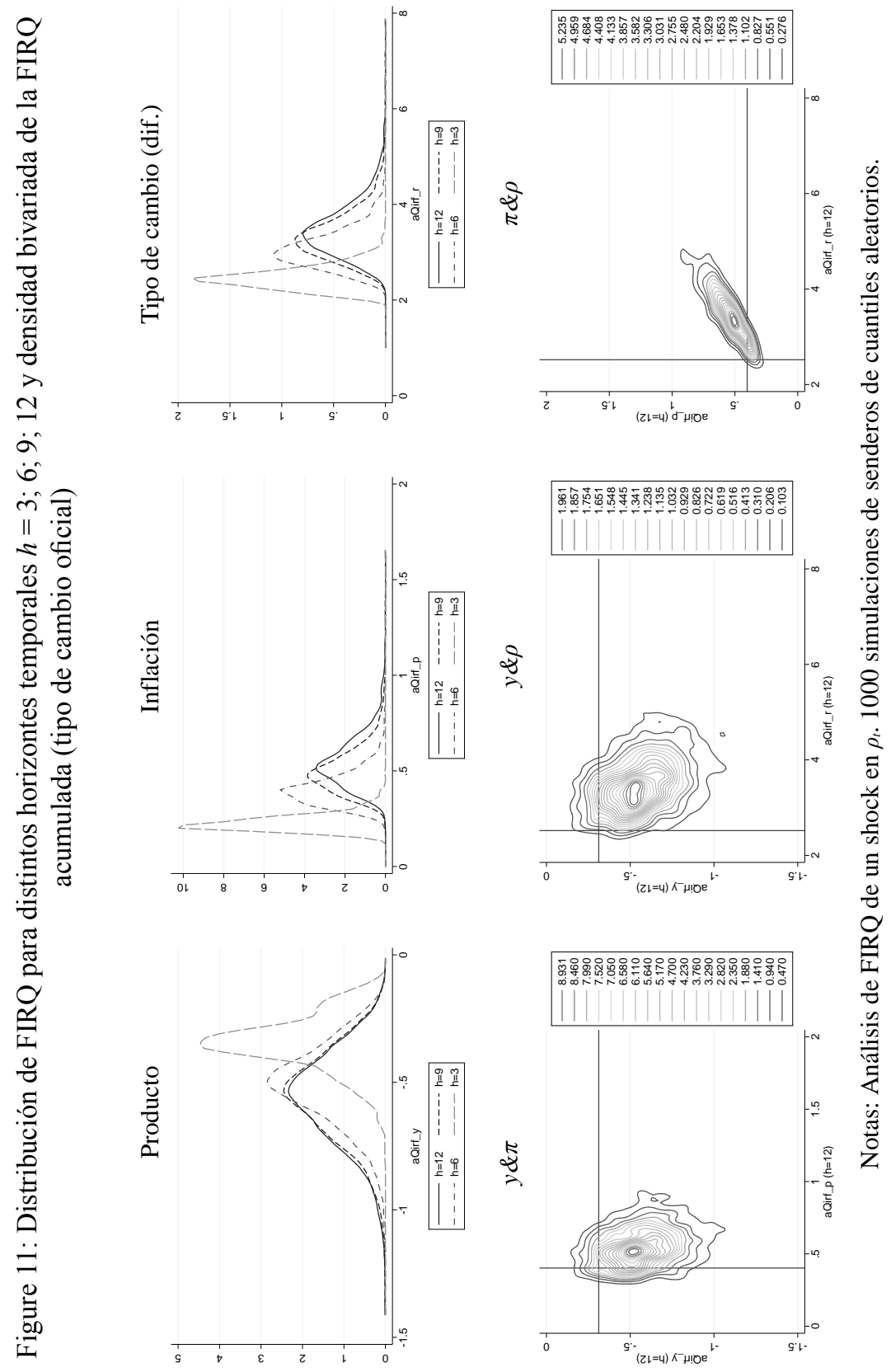


UNA EVALUACIÓN DEL PASS-THROUGH EN LA ARGENTINA USANDO FUNCIONES...

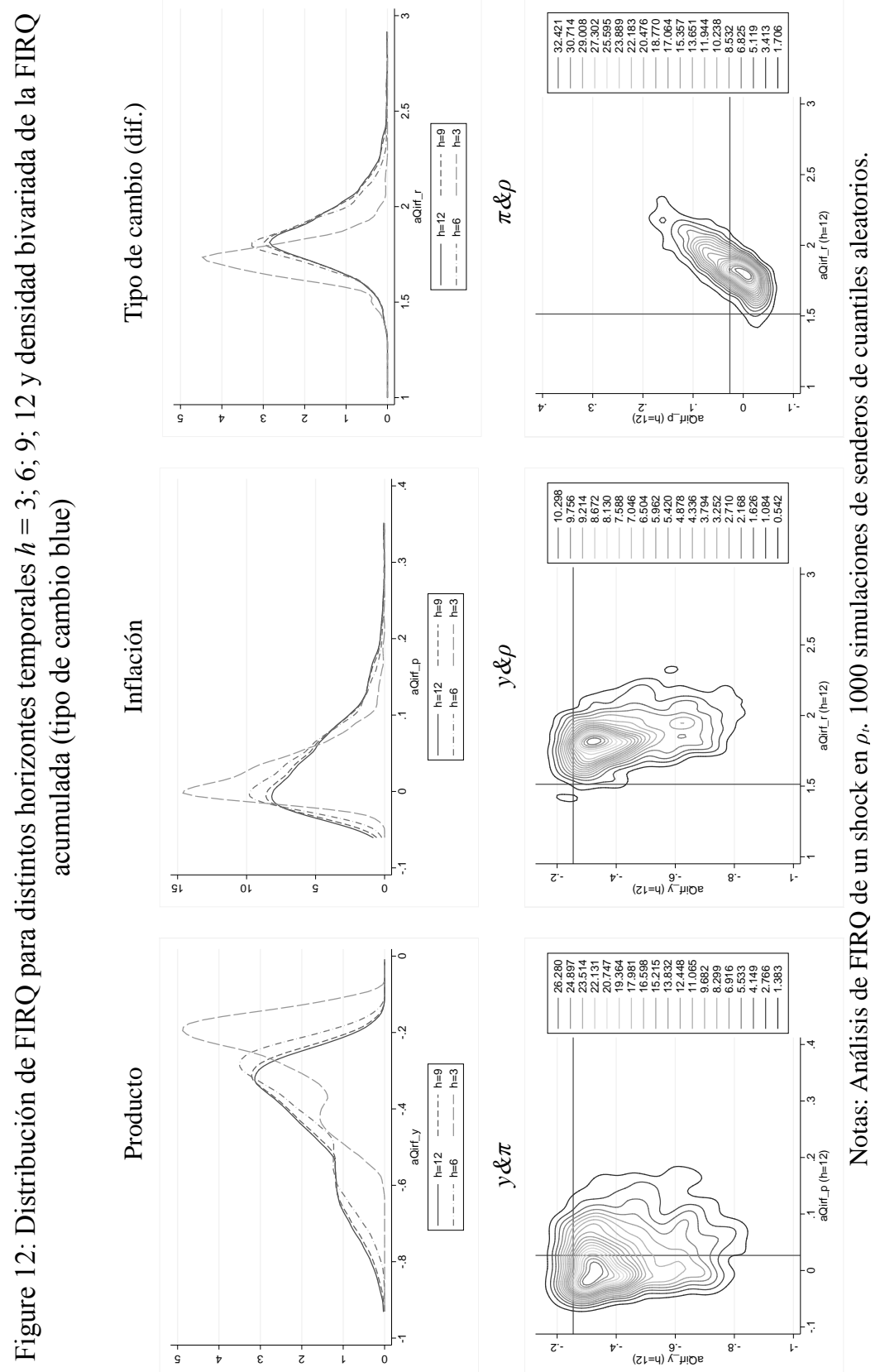


$\mathrm{h}=1,2, \ldots, 12$. Así podemos evaluar las distintas posibilidades de ocurrencia de las aFIRQ, es decir los efectos acumulados, luego de un mismo shock (que siempre corresponde a un shock en el tipo de cambio, en diferencias). En particular, nos interesa la distribución para $h=3,6,9,12$ y especialmente para $h=12$, que puede considerarse como el largo plazo. Las figuras 11 y 12 reportan estas distribuciones estimando en cada caso las densidades con métodos de kernel, para los modelos de tipo de cambio oficial y blue, respectivamente. Los gráficos de densidades univariadas se corresponden con la distribución de las funciones aFIRQ para las tres variables endógenas, cada una por separado. Estos gráficos permiten evaluar la heterogeneidad específica en los efectos de la FIR en cada variable. Las densidades bivariadas estiman las densidades bivariadas de a pares (producto-inflación, producto-tipo de cambio, inflación-producto) para $\mathrm{h}=12$ solamente (doce meses hacia adelante, que se considera el largo plazo). Estos gráficos ilustran las interacciones entre la ocurrencia de distintos valores de la aFIRQ en una variable y en otra.

Los efectos de mediano y largo plazo presentan una amplia variedad de resultados posibles. Esto justamente nos da una cuantificación de la heterogeneidad en los efectos de pass-through luego de un shock cambiario. Este análisis permite entonces evaluar qué posibles escenarios podemos tener luego de un shock determinado. De particular interés es la ocurrencia de un escenario de gran recesión y alta inflación, que ocurre con mucha más frecuencia de la que hubiera sido esperada con el modelo en la media solamente.

Sobre la base de las densidades bivariadas de producto e inflación podemos ver que a mayor efecto sobre la inflación (mayor pass-through a precios) mayor el efecto recesivo. De hecho, estos efectos están asociados con una alta persistencia en el efecto del shock cambiario sobre sí mismo. Podemos entonces inferir que una devaluación que no se propaga en mayor devaluación futura es menos recesiva que una que tiene alta persistencia.

\section{CONCLUSIÓN}

Dada la importancia que tiene el efecto pass-through de tipo de cambio a precios y producto, este trabajo contribuye a evaluar la heterogeneidad de dichos efectos usando cuantiles multivariados. Los efectos dinámicos computados revelan la existencia de heterogeneidad en los traslados a precios y producto.

Este trabajo muestra que un shock cambiario puede tener distintos efectos sobre la inflación y el producto. Los siguientes son los principales resultados. 
En primer lugar, los efectos FIR de la media, calculados con un modelo VAR en la media, no resultan adecuados para el producto y la inflación. De hecho los efectos calculados de esta manera se condicen con cuantiles extremos en el análisis de FIRQ. Esto implica que pueden ocurrir eventos (por ejemplo, alta inflación) con cierta probabilidad positiva que no son capturados a partir del análisis en la media.

Segundo, los efectos de pass-through usando el tipo de cambio oficial sobre el producto y la inflación dependen mayormente de los cuantiles de cada variable y no parece haber efectos cruzados. Es decir, los efectos sobre el producto varían en cuanto a los cuantiles del producto pero no de la inflación. En el mismo sentido, los efectos sobre la inflación no dependen de los cuantiles del producto y sí de la misma inflación. Una implicancia práctica de este resultado es que el nivel de pass-through a precios no depende de si nos encontramos en una fase recesiva o expansiva del producto. En términos del pass-through a precios se observa que el traslado es mayor (menor) para menores (mayores) cuantiles de la inflación. Existen efectos cruzados, sin embargo, cuando usamos el tipo de cambio blue. Los cuantiles del producto afectan el pass-through a precios: períodos recesivos (expansivos) tienen un pass-through más alto (bajo). Los cuantiles de la inflación explican la heterogeneidad en la respuesta del producto: períodos de alta inflación producen un efecto más negativo en el producto ante una devaluación.

Tercero, tanto el producto como la inflación dependen de los cuantiles del tipo de cambio. Esto significa que distintas dinámicas del tipo de cambio puede producir dinámicas de alto pass-through (negativo para el producto, positivo para la inflación). El resultado final del trabajo es la representación gráfica de la gama de posibilidades de ocurrencia de distintos efectos luego de un mismo shock cambiario. Los gráficos muestran que eventos extremos de alta inflación y/o severa recesión son probables y deberían ser tenidos en cuenta.

\section{REFERENCIAS BIBLIOGRÁFICAS}

Aron, J., Macdonald, R., \& Muellbauer, J. (2014). Exchange rate pass-through in developing and emerging markets: A survey of conceptual, methodological and policy issues, and selected empirical findings. Journal of Development Studies, 50 (1), 101-143.

Barhoumi, K. (2006). Differences in long run exchange rate pass-through into import prices in developing countries: An empirical investigation. Economic Modelling, 2 (6), 926-951. 
Beirne, J., \& Bijsterbosch, M. (2011). Exchange rate pass-through in central and eastern European EU Member States. Journal of Policy Modeling, 33 (2), 241-254.

Ozkan, I., \& Erden, L. (2015). Time-varying nature and macroeconomic determinants of exchange rate pass-through. International Review of Economics and Finance, $38(\mathrm{C}), 56-66$.

Borensztein, E., \& Queijo von Heideken, V. (2016). Exchange rate pass-through in South America: An overview. IDB Working Paper Series No. 710.

Ca'Zorzi, M., Hahn, E., \& Sánchez, M. (2007). Exchange rate pass-through in emerging markets. The IUP Journal of Monetary Economics, 5 (4), 84-102.

Carlier, G., Chernozhukov, V., \& Galichon, A. (2016). Vector quantile regression. Annals of Statistics, 44 (3), 1165-1192.

Caselli, F. G., \& Roitman, A. (2016). Non-linear exchange rate pass-through in emerging markets. IMF Working Paper No. 16/1.

Castiglione, B. (2017). El traspaso a precios de las depreciaciones cambiarias: una estimación VECM para el caso argentino (2005-2017). Recuperado de https://www.bcra.gob.ar/institucional/DescargaPDF/DownloadPDF. aspx? Id=606.

Choudhri, E., \& Hakura, D. (2006). Exchange rate pass-through to domestic prices: Does the inflationary environment matter? Journal of International Money and Finance, 25 (4), 614-639.

Christiano, L., Eichenbaum, M., \& Evans, C. (1996). The effects of monetary policy shocks: Evidence from the flow of funds. Review of Economics and Statistics, 78 (1), 16-34. DOI: $10.2307 / 2109845$

Edwards, S. (2006). The relationship between exchange rates and inflation targeting revisited. NBER Working Paper No. 12163.

Galvao, A., Montes-Rojas, G., \& Park, S-Y. (2013). Quantile autoregressive distributed lag model with an application to house price returns. Oxford Bulletin of Economics and Statistics 75 (2), 307-321.

Ghartey, E. (2018). Asymmetries in exchange rate pass-through and monetary policy principle: Some Caribbean empirical evidence. North American Journal of Economics and Finance, 47, 325-335.

Jiménez, R., \& Morales, A. (2016). A new look at exchange rate pass-through in the G-7 countries. Journal of Policy Modeling, 38 (5), 985-1000.

Law, K., Satoh, E., \& Yoshimi, T. (2019). Exchange rate pass-through at the individual product level: Implications for financial market integration. North American Journal of Economics and Finance, 46 (C), 261-271.

Hallin, M., Paindaveine, D., \& Siman, M. (2010). Multivariate quantiles and multiple output regression quantiles: From L1 optimization to halfspace depth. Annals of Statistics, 38 (2), 635-669. 
Koenker, R., \& Xiao, Z. (2006). Quantile autoregression. Journal of the American Statistical Association, 101 (475), 980-990.

McCarthy, J. (2007). Pass-through of exchange rates and import prices to domestic inflation in some industrialized economies. Eastern Economic Journals, 33 (4), $511-537$.

Menon, J. (1995). Exchange rate pass-through. Journal of Economic Surveys, 9 (2), $197-231$.

Miller, S. (2003). Estimación del pass-through del tipo de cambio a precios: 19952002. Revista Estudios Económicos, 10. Recuperado de http://suscripciones. bcrp.gob.pe/docs/Publicaciones/Documentos-de-Trabajo/2003/Documento-Trabajo-05-2003.pdf

Montes-Rojas, G. (2017). Reduced form vector directional quantiles. Journal of Multivariate Analysis, 158 (C), 20-30.

Montes-Rojas, G. (2019). Multivariate quantile impulse response functions. Journal of Time Series Analysis, 40 (5), 739-752.

Paindaveine, D., \& Siman, M. (2011). On directional multiple-output quantile regression. Journal of Multivariate Analysis, 102 (2), 193-212.

Paindaveine, D., \& Siman, M. (2012). Computing multiple-output regression quantile regions. Computational Statistics and Data Analysis, 56 (4), 840-853.

Palleja, M. J. (2017). Traspaso condicional de tipo de cambio a precios. Trabajo presentado en la XLI Reunión Anual de la Asociación Argentina de Economía Política. AAEP, San Carlos de Bariloche.

Ramey, V. (2016). Macroeconomic shocks and their propagation. En J. B. Taylor \& H. Uhlig (Eds.), Handbook of Macroeconomics, Vol. 2, (pp. 71-162). North-Holland, Amsterdam: Elsevier.

Stock, J., \& Watson, M. (2016). Dynamic factor models, factor-augmented vector autoregressions, and structural vector autoregression in macroeconomics. En J. B. Taylor \& H. Uhlig (Eds.), Handbook of Macroeconomics, Vol. 2 (pp. 415-525). North-Holland, Amsterdam: Elsevier.

Taylor, J. (2000). Low inflation, pass-through and the pricing power of firms. European Economic Review, 44 (7), 1389-1408.

(C) 2019 por los autores; licencia otorgada a la revista Estudios económicos. Este artículo es de acceso abierto y distribuido bajo los términos y condiciones de una licencia Atribución-No Comercial 3.0 Unported (CC BY-NC 3.0) de Creative Commons. Para ver una copia de esta licencia, visite http://creativecommons.org/ licenses/by-nc/3.0/ 
